\title{
On the Cross Correlation Properties of MIMO Wideband Channels under Nonisotropic Propagation Conditions
}

\author{
Ana Maria Pistea ${ }^{1}$ and Hamidreza Saligheh $\operatorname{Rad}^{2}$ \\ ${ }^{1}$ Military Technical Academy, Bucharest, 050141 Bucharest, Romania \\ ${ }^{2}$ Tehran University of Medical Sciences, Tehran 16656, Iran \\ Correspondence should be addressed to Hamidreza Saligheh Rad; hamid.saligheh@gmail.com
}

Received 20 August 2014; Accepted 12 December 2014

Academic Editor: Xinyi Tang

Copyright (c) 2015 A. M. Pistea and H. Saligheh Rad. This is an open access article distributed under the Creative Commons Attribution License, which permits unrestricted use, distribution, and reproduction in any medium, provided the original work is properly cited.

\begin{abstract}
Wideband (WB) and ultrawideband (UWB) systems combined with multiple-input multiple-output (MIMO) technology increase both the systems performances and the complexity of the channel models required to evaluate their capabilities. Because in real scenarios waves propagate nonisotropically, the accuracy of the channel model is increased if nonisotropic propagation is considered. The channel bandwidth is the key term in the evaluation process of these systems because large bandwidths introduce frequency selectivity, a unique phenomenon of WB and UWB systems with more complexity in the latter case. This is due to the fact that, unlike WB technology in which the propagating signal is the only affected parameter by the frequency selectivity, in the UWB case, this phenomenon also affects the antenna propagation pattern (APP). In this paper, we developed a novel channel model based on the statistical analysis of two-dimensional cross correlation functions (CCFs) of WB/UWB MIMO nonisotropic channels. A mathematical solution to assess the frequency selective behavior of the UWB APP is also presented. The CCF reveals how the power spectral density (PSD) of the channel is influenced by bandwidth, nonisotropic propagation, and APP.
\end{abstract}

\section{Introduction}

The design of high quality wideband (WB) and ultrawideband (UWB) multiple-input multiple-output (MIMO) wireless systems requires the development of two-dimensional (2D) space-time-frequency (STF) channel models along with the evolution from narrowband (NB) to WB/UWB transmissions. This evolution from NB to WB/UWB MIMO channels brings new and more complex propagation phenomena involving the following open questions.

(1) What are the main propagation phenomena that appear in a $2 \mathrm{D}$ propagation scenario when the channel bandwidth increases?

(2) In a $2 \mathrm{D}$ scenario, how does the influence of the antenna propagation pattern (APP) on NB channels differ from the influence of the APP on WB/UWB channels characteristics?

(3) Is a 2D channel model developed for the study of WB channels adequate for the analysis of UWB channels?
An exhaustive approach to characterize WB/UWB MIMO channels and to answer the questions enumerated above is to statistically analyze the behavior of their space-timefrequency (STF) channel transfer functions (CTFs). We propose a model for both WB and UWB MIMO channels based on two-dimensional (2D) STF cross correlation function (CCF) between the CTFs of two subchannels of a multicarrier (orthogonal frequency division multiplexing, OFDM) channel. A subchannel represents the connection between two antennas, one at the base station (BS) and the other at the mobile station (MS), which transmit/receive the signal at specific time and frequency.

The calculation of the CCF for WB/UWB MIMO channels has attracted the attention of several researchers, for example, [1-5]. Ma and Pätzold extended an NB MIMO channel model to a version adequate for WB channels analysis. The main improvement made to extend the applicability of the NB model was to add the elements which introduce the frequency selectivity caused by the propagation channel. This model presents the 2D CCF derived only for isotropic propagation 
[1]. Pätzold and Hogstad developed a WB MIMO channel model with temporal, spatial, and frequency correlation properties. This model presents the 2D CCF derived for both isotropic and nonisotropic propagation assuming a specific geometry of scatterers around receiver [2]. Saleh and Valenzuela $(\mathrm{S}-\mathrm{V})$ developed one of the first UWB channel models which was proposed to reproduce the multipath effect of indoor environments. Using S-V results, Zou et al. determined a CCF expression of multiband OFDM-UWB discrete-time base-band channel impulse response $[3,4]$. Abdi and Kaveh propose an ST-CCF for a MIMO frequency nonselective Rician fading channel, assuming a one ring of scatterers model around the MS [5]. This model is as an example of an outdoor environment, where the von-Mises probability density function (PDF) is suggested to model the nonisotropic propagation environment around the user.

As a summary, our literature review shows that the general approach is to develop channel models for WB transmissions separated from the channel models for UWB communications. Another trend is to include the effects of the propagation environment (waves clustering, frequency selectivity) and to ignore the frequency selective behavior of the antenna patterns. Because UWB systems were mainly developed for indoor applications the research in the field of UWB outdoor channel modeling was not a priority [68]. From the literature review, it was also observed that most of the MIMO-CCF models are based on a specific geometry for scatterers distribution around MS to characterize the nonisotropic propagation.

Lately, UWB technology in combination with MIMO systems finds more and more interest in areas such as location tracking application for outdoor emergency services $[9,10]$, location tracking and sensor applications for mobile outdoor users [11], and medical [12] and electronic warfare applications [13]. Even more, some research results [14-16] proved that a joint radar and wireless communication system would constitute a unique platform for future intelligent environmental sensing and ad hoc communication networks, in terms of both spectrum efficiency and cost effectiveness. This evolution regarding the use of UWB systems in outdoor environments, as radar or communication system, has led to the formulation of specific requirements aiming at investigating the influence of outdoor propagation on UWB mobile systems $[17,18]$.

The model proposed in this paper describes the CCF between two subchannels of an outdoor WB/UWB MIMO channel employing directional and omnidirectional antennas and in the presence of nonisotropic wave propagation in $2 \mathrm{D}$ space. Instead of assuming a specific geometry for scatterers in space (models based on specific geometries of scatterers in the space are just able to predict the behavior of that particular propagation scenario), we used specific mathematical relationships between the physical parameters of the wireless channel along with appropriate assumptions on their PDFs to derive a channel model based on the CCF of outdoor WB and UWB MIMO channels. This model is an extension of a 2D NB channel model [19]. The evolution through systems with (ultra)wide bandwidths implies new propagation effects that make the NB model inappropriate for $\mathrm{WB} / \mathrm{UWB}$ transmissions. In order to extend the NB channel model to a version adequate for WB/UWB channels analysis, the mathematical structure of the model needs to be updated with the elements which characterize the new propagation phenomena (represented by channel frequency selectivity, waves clustering) and with the elements which define the performances of the new systems (represented by operating bandwidth, frequency selectivity of the antenna). We represent the nonisotropic scatterers by the Fourier series expansion (FSE) of the PDF of the propagating directions. We also consider the effect of directional and omnidirectional antenna element patterns by the FSE of the APPs. The expression of the CCF turns out to be a composition of linear expansions of Bessel functions of the first kind. Fourier analysis of the derived CCF is used to determine the power spectral density (PSD) of WB and UWB channels in a stationary scenario. The mathematical set-up of the stationary scenario transforms the MIMO channel into a multiple-input single-output (MISO) channel to be analyzed as a special case. The contribution in this 2D-CCF model is fourfold.

(1) It includes the main propagation phenomena that appear in 2D WB/UWB MIMO outdoor scenarios and permits the evaluation of combined effects of channel bandwidth, nonisotropic scattering, APP (omnidirectional or directional), and the array interelement distance on the CCF of WB and UWB MIMO channels.

(2) This model can be used for the analysis of both WB and UWB propagation channels. The key to apply a model for the analysis of both types of channels is to use the appropriate expressions for APPs. Since WB APP is not frequency selective, the relative bandwidth being a small fraction of the central frequency and the UWB APP is frequency selective and different antenna patterns expressions for these two types of systems should be used. In this paper we propose mathematical expression for UWB APP.

(3) It accurately reflects the influence of the MS direction (and speed) on the spectral characteristics of WB and UWB channels.

(4) It gives mathematical expressions to evaluate the channel power spectrum of WB and UWB wireless channels.

In [20] a three-dimensional (3D) version of this model was presented. The difference between $2 \mathrm{D}$ and $3 \mathrm{D}$ versions of the proposed model consists of APP and nonisotropic propagation representations. In the case of the $2 \mathrm{D}$ model the APP and the propagation environment are represented depending on azimuth angle spread (AAS). The 3D version of the model represents these two elements as a function of both AAS and elevation angle spread (EAS). From the obtained results it was observed that the $2 \mathrm{D}$ model provides a good representation of how the frequency selectivity affects the signal spectrum. A 2D model for most propagation environments is more eligible when we get closer to the ground because a propagation environment behaves similar 
to a $2 \mathrm{D}$ environment. Another advantage is that such model represents the propagation environment fairly accurately, with minimum complexity and difficulty. The model presented in this paper is an extension of the conference paper presented in [21]. There are significant differences between the results presented in [21] and the content of the current work, where both the CCF and the PSD are derived and evaluated for WB/UWB MIMO channels while [21] describes only the PSD of WB/UWB systems in a stationary case for MISO channels. The latter paper is mostly about PSD, the derivation process of the CCF (which is the root of the PSD) was not presented, and the model cannot be extended to be used in other scenarios (e.g., indoor propagation, MIMO channels). This statement is also valid for [20] which does not contain the derivation process of the three-dimensional CCF. In the present work are provided details regarding the calculation of the moment generating functions (MGFs$\left.\Phi_{\tau, s}\right)$ of the delay profile $(\tau, T)$ and details regarding the calculation of the number of Fourier series coefficients (FSCs) for different PDFs of the AAS necessary to approximate Laplace and von-Mises distributions and how they are further involved in assessing the CCF. Since in $[20,21]$ CCF was not derived and graphically represented this connection between FSCs, APP, distribution of the propagation environment and CCF behavior cannot be established.

The rest of this paper is organized as follows: in Section 2, the notations and the parameters included in the 2D WB/UWB channels model are described; in Section 3, the $\mathrm{WB} / \mathrm{UWB} \mathrm{CCF}$ expression is derived and is numerically evaluated. In Section 4, the Fourier analysis of the CCF which results in the PSD of the WB and UWB channels is presented; in Section 5, conclusions are presented.

\section{Two-Dimensional Wideband/UWB MIMO Model Description}

In this section, we describe the propagation scenario, the notations used throughout this paper, and the elements (accompanied by their mathematical expressions) introduced in the structure of the CTF valid for WB and UWB channels. We also emphasize the differences which exist between WB and UWB channel models and how these differences are included in our model.

By definition, a channel is considered WB if the fractional bandwidth $\left(B_{f}\right)$ (the ratio of bandwidth at $-10 \mathrm{~dB}$ points to center frequency) is $1 \%<B_{f} \leq 20 \%$ while UWB channels are characterized by $20 \%<B_{f} \leq 200 \%$ [22-24]. For both, WB and UWB scenarios, we consider a moving MS with a constant speed vector $V(\mathrm{~m} / \mathrm{sec})$ and a fixed BS in a $2 \mathrm{D}$ nonisotropic propagation environment. The resulting CTF is determined by the $p$ th transmitting antenna element at the $\mathrm{BS}$ side, the propagation environment, and the $m$ th receiving antenna element at the MS. CTF description is based on two categories of elements: $(\mathrm{C} 1)$ elements assigned to the communication system, which are the system bandwidth and APPs at the BS and at the MS, and (C2) elements assigned to the wireless channel represented by nonisotropic propagation, frequency selectivity, and clustering phenomenon.
Both categories of elements enumerated above changed their mathematical representation once with the evolution from $\mathrm{NB}$ to $\mathrm{WB}$ and UWB channels as it is presented in the following lines.

(C1) The APPs of the $p$ th and $m$ th antenna of the BS and the MS array, $G_{p}^{B}\left(\Theta^{B}, \omega\right), G_{m}^{M}\left(\Theta^{M}, \omega\right)$, give the response of the antenna in terms of the propagation directions, $\Theta$, and frequency, $\omega$. These functions implicitly include the effect of mutual coupling caused by the neighboring antenna elements. Are all periodic functions of $2 \pi$, where $\Theta^{B}$ - and $\Theta^{M}$ are unity vectors which represent a propagation direction (DOD or DOA) at BS or MS, respectively. We assume that the DOA (to the receiver) and the DOD (from the transmitter) are independent. Therefore, we represent them by their FSCs as follows:

$$
G(\Theta ; \omega)=\sum_{k=-\infty}^{\infty} \mathscr{G}_{k} e^{j k \Theta}, \quad \mathscr{G}_{k}=\frac{1}{2 \pi} \int_{-\pi}^{\pi} G(\Theta ; \omega) e^{-j k \Theta} d \Theta .
$$

For NB channels, it is assumed that the response of the antenna does not change significantly over the bandwidth since the relative bandwidth is a small fraction of the central frequency [19]. This assumption is also true for WB channels but proved to be false once with the evolution through ultrawide bandwidths. UWB antenna patterns are frequency selective and this characteristic should be considered in the design process of UWB channel models. The radiation pattern depicts the relative strength distribution of the transmitted or received power by the antenna. In [25, page 19] it is suggested that, in order to correctly evaluate the received and the transmitted power of UWB signals, the radiation pattern should be determined across the entire frequency spectrum. This requirement is the consequence of the frequency selectivity phenomenon. Thus, according to the method suggested in [25], depending on the signal bandwidth, we propose two approaches for 2D APP calculation as follows:

(a) for WB signals, APP is calculated depending on the central angular frequency, $\omega$;

(b) for UWB signals, we calculate APP depending on the central frequency and by integrating $G(\Theta, \omega)$ across all the frequencies of the transmitted signal.

Table 1 presents the APPs of two WB/UWB antennas. The helical (directional) and rectangular (omnidirectional) antennas are often used for antenna arrays and (ultra)wide bandwidth applications [22].

$f_{H}, f_{L}$ are the upper and the lower frequencies of the UWB channel bandwidth, the parameter $h$ is proportional with the size of the antenna, and $G_{0}$ is the real and positive constant antenna gain that varies for each antenna.

(C2) The nonisotropic propagation is characterized by the nonuniform distribution of the waveforms around the MS and the BS in space. In order to describe a nonisotropic channel the PDF of the propagating directions, known as azimuth angular spread (AAS), can be used. In the proposed model, the PDF of the propagation directions is denoted by $f^{B}\left(\Theta^{B}\right)$, at the BS, and $f^{M}\left(\Theta^{M}\right)$, at the MS. One candidate for 
TABLE 1: $2 \mathrm{D}$ antenna radiation patterns.

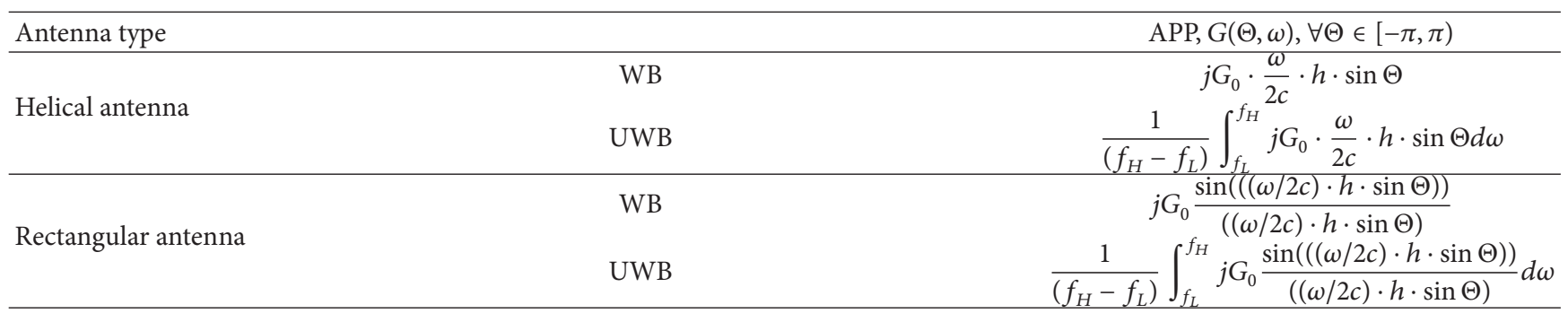

TABLE 2: Nonisotropic AAS and corresponding Fourier series coefficients.

\begin{tabular}{l}
\hline PDF, $f_{\Theta}(\Theta), \forall \Theta \in[-\pi, \pi), \mathscr{F}_{k}$ \\
Laplace \\
$\qquad \mathscr{F}_{\Theta}(\Theta)=\frac{e^{|((-\sqrt{2} \Theta) / \sigma)|}}{\sqrt{2} \sigma}$ \\
Von-Mises \\
$f_{\Theta}(\Theta)=\frac{e^{-((\pi(\sqrt{2}+j k \sigma)) / \sigma)}\left(e^{((2 \sqrt{2} \pi) / \sigma)}-e^{j 2 k \pi}\right)}{2 \pi(j \sqrt{2} k \sigma-2)}$ \\
$\mathscr{F}_{k}=\frac{J_{k}(n)}{J_{0}(n)}$
\end{tabular}

the PDF of the nonisotropic AAS is Laplace distribution [26]. Another distribution which characterizes the nonisotropic environment is the von-Mises PDF [27]. Since these PDFs are periodic functions with period $2 \pi$, in Table 2 we represent them by the corresponding FSC:

$$
f_{\Theta}(\Theta)=\sum_{k=-\infty}^{+\infty} \mathscr{F}_{k} e^{j k \Theta}, \quad \mathscr{F}_{k}=\frac{1}{2 \pi} \int_{-\pi}^{\pi} f_{\Theta}(\Theta) e^{-j k \Theta} d \Theta .
$$

Von-Mises PDF is strongly influenced by parameter $n$, which determines the order of the channel nonisotropy. In other words $n$ controls the width of DOA of scattered components. This parameter can take values in the range $n \in$ $[0, \infty)$. When $n \rightarrow \infty, f_{\Theta^{M}}(\Theta)=\delta(\Theta-\mu)$, the propagation environment is considered extremely nonisotropic-scattered concentrated at $\Theta=\mu$, where $\mu \in[-\pi, \pi)$ is the mean DOA at the MS. For large $n$, say $3 \leq n \leq 20$, we have a typical nonisotropic environment [27]. When FSCs are determined this parameter appears in the argument of the Bessel functions represented by the modified Bessel function of the first kind, $J_{k}(\cdot)$, and the zero-order modified Bessel function, $J_{0}(\cdot)$.

The isotropic propagation environment can be mathematically represented in a similar way, using the following expression of the FSC: $\mathscr{F}_{k}=(1 / 2 \pi) \delta_{k}$. Laplace and von-Mises distributions are specific to WB/UWB MIMO channels.

The complexity of the nonisotropic propagation environment is increased by two phenomena which appear only in the case of WB and UWB channels.

Clustering Phenomenon. NB systems receive most of the multipath components (MPCs) within the symbol duration.
In these circumstances, the receiver acts as if there was a single multipath component. Due to the better time resolution of WB and UWB systems the multipath components tend to arrive in cluster, rather than in a continuum as it is common for NB channels. The authors of [28] define a cluster as an accumulation of MPCs with similar time of arrivals and angle of arrivals. As a consequence of this phenomenon, the CTF of $\mathrm{WB} / \mathrm{UWB}$ channels is the summation of the dominant $I$ paths and $L$ clusters. In the case of NB channels the CTF appeared to be only the summation of $I$ paths.

The propagation delay of the $i$ th path within the $l$ th cluster between the pth (at the BS) and $m$ th (at the MS) antenna elements $\tau_{p m, i l}$ is decomposed into three components: one component which represents the delay depending on the distance between BS and MS and other two components which vary as a function of the local coordinates of BS and MS:

$$
\begin{gathered}
\tau_{p m, i l}=\tau_{i l}-\left(\tau_{p, i l}^{B}+\tau_{m, i l}^{M}\right), \\
\tau_{p, i l}^{B} \triangleq \frac{a_{p}^{B} \Theta_{i l}^{B}}{c} ; \quad \tau_{m, i l}^{M} \triangleq \frac{a_{m}^{M} \Theta_{i l}^{M}}{c},
\end{gathered}
$$

where $(\cdot)^{T}$ represents the transpose operator, $\tau_{i l}$ is the delay between local coordinates $O^{B}$ or $O^{M}$, and $\tau_{p, i l}^{B}, \tau_{m, i l}^{M}$ represent the propagation delays from antenna $a_{p}^{B}$ to $a_{m}^{M}$ located in their corresponding coordinates $O^{B}$ or $O^{M} . T_{l}$ is the cluster arrival rate and is considered to be constant in time. $\Theta_{i l}^{B}$ and $\Theta_{i l}^{M}$ were previously defined.

Frequency Selectivity Phenomenon. This refers to the different attenuation that the signal subbands undergo. In our model, the frequency selectivity of the radio channel is characterized by two components. These components are represented by APP (valid only for UWB channels) and by the term $\left(\omega_{b w} / \omega\right)^{\eta}$. The parameter $\omega_{b w}$ represents the signal bandwidth $\omega_{b w}=\omega_{H}-\omega_{L} ; \omega_{L}$ and $\omega_{H}$ are the lower and the upper angular frequencies. $\eta$ depends on the geometric configuration of the objects which produce the signal's diffraction. The following values can be assigned to $\eta$ : -1 (diffraction by corner or tip), 0.5 (diffraction by axial cylinder face), and 1 (diffraction by broadside of a cylinder) [29]. When modeling NB channels, it was adequate to define the path gain depending on the path time-delay [19]. This is not sufficient for WB/UWB MIMO channels 
where the frequency selectivity phenomenon influences the gain of the channel. In the ray based propagation models which can be applied to signals transmitted at high frequency ranges, like WB and UWB signals, it can be assumed that one propagation path has DOA and time of arrival that does not depend on frequency but has a frequency dependent complex path gain. In our model the path gain of the $i$ th path within the $l$ th cluster which propagates from the $p$ th antenna to the $m$ th antenna is expressed as the extension of Friis' transmission formula $g_{p m, i l}=1 / 2 \omega \tau_{i l}$.

When putting together the elements described in $(\mathrm{Cl})$ and (C2), the CTF of WB and UWB channels results in the following expression:

$$
\begin{aligned}
& h_{p m}(t, \omega) \\
&=\left(\frac{\omega_{b w}}{\omega}\right)^{\eta} \sum_{l=1}^{L} \sum_{i=1}^{I} G_{p}^{B}\left(\Theta_{i l}^{B} ; \omega\right) \\
& \quad \times G_{m}^{M}\left(\Theta_{i l}^{M} ; \omega\right) g_{p m, i l} e^{j\left(\phi_{i l}-\overline{\omega_{i l}} t-\omega \tau_{i l}-\omega T_{l}\right)} .
\end{aligned}
$$

The resulting CTF is represented by the double summation of clusters, $l$, and of the propagation waveforms, $i$, over a maximum number of clusters, $L$, and paths, $I$. Within each cluster (path) the signal reaches the receiver with a response described by the PDFs of some random variables. These random variables are phase, delay, DOD, and DOA. In the resulting CTF each $l$ cluster and implicitly each $i$ wave are associated with a path attenuation gain, $g_{p m, i}$, a path phase shift, $\phi_{i l}$, and a time-varying delay, $\tau_{p m, i l}(t) \triangleq \tau_{p m, i l}+$ $(t / c) V^{T} \Theta_{i l}^{M}$. The Doppler shift, of the $i$ th received wave within the $l$ th cluster, is represented by $\overline{\omega_{i l}}=(\omega / c) V^{T} \Theta_{i l}^{M}$ where $V$ and $c$ are the MS velocity vector and the speed of light, respectively.

\section{Two-Dimensional Cross Correlation Function of WB/UWB MIMO Channels}

The CCF expression of the CTFs, $h_{p m}\left(t_{1}, \omega_{1}\right)$ and $h_{q n}\left(t_{2}, \omega_{2}\right)$, of two arbitrary subchannels of a MIMO channel is the result of the following definition:

$$
R_{p m, q n}\left(t_{1}, t_{2}, \omega_{1}, \omega_{2}\right) \triangleq E\left[h_{p m}\left(t_{1}, \omega_{1}\right) h_{q n}^{*}\left(t_{2}, \omega_{2}\right)\right] .
$$

In the CCF expression there are three dimensions: space, two pairs of antenna elements $(p, m, q, n)$, time $\left(t_{1}, t_{2}\right)$, and central frequencies $\left(\omega_{1}, \omega_{2}\right)$. According to these three dimensions, we call it STF-CCF. The expectation operation is performed over all introduced random variables. In the presence of enough number of multipaths by invoking the central limit theorem the CTF can be considered a Gaussian random process. Therefore, the above second-order statistics fully characterize statistical behavior of the channel. By replacing (4) with (5), the CCF results in the following expression:

$$
\begin{aligned}
& R_{p m, q n}\left(t_{1}, t_{2}, \omega_{1}, \omega_{2}\right) \\
& =\frac{\left(\omega_{b w 1} \omega_{b w 2}\right)^{\eta}}{\left(\omega_{1} \omega_{2}\right)^{\eta}} \\
& \times \sum_{l=1}^{L} \sum_{i=1}^{I}\left\{E \left[G_{p}^{B}\left(\Theta_{i_{1} l_{1}}^{B} ; \omega_{1}\right) G_{m}^{M}\left(\Theta_{i_{1} l_{1}}^{M} ; \omega_{1}\right)\right.\right. \\
& \times g_{p m, i_{1} l_{1}} \times g_{q n, i_{2} l_{2}} \\
& \left.\times e^{\left(-j \omega_{1} T_{p m, l_{1}}-j \omega_{1} \tau_{p m, i_{1} l_{1}}\left(t_{1}\right)\right)}\right] \\
& \times E\left[G_{q}^{B^{*}}\left(\Theta_{i_{2} l_{2}}^{B} ; \omega_{2}\right) G_{n}^{M^{*}}\left(\Theta_{i_{2} l_{2}}^{M} ; \omega_{2}\right)\right. \\
& \times e^{j\left(\phi_{i_{1} l_{1}}-\phi_{i_{2} l_{2}}\right)} \\
& \left.\left.\times e^{\left(-j \omega_{2} T_{q n, l_{2}}-j \omega_{2} \tau_{q n, i_{2} l_{2}}\left(t_{2}\right)\right)}\right]\right\} .
\end{aligned}
$$

Regrouping dependent and independent random variables and using the elements described in (C1) and (C2) we obtain

$$
\begin{aligned}
& R_{p m, q n}\left(t_{1}, t_{2} ; \omega_{1}, \omega_{2}\right) \\
& =\frac{\left(\omega_{b w 1} \omega_{b w 2}\right)^{\eta}}{\left(\omega_{1} \omega_{2}\right)^{\eta}\left(4 \omega_{1} \omega_{2}\right)} \\
& \times\left\{\sum _ { l = 1 } ^ { L } \sum _ { i = 1 } ^ { I } E \left[\left(\tau_{i_{2} l_{2}} \tau_{i_{1} l_{1}}\right)^{-1}\right.\right. \\
& \left.\times e^{j\left(\left(\omega_{2} \tau_{i_{2} l_{2}}-\omega_{1} \tau_{i_{1} l_{1}}\right)+\left(\omega_{2} T_{l_{2}}-\omega_{1} T_{l_{1}}\right)\right)}\right] \\
& \times E\left[e^{j\left(\phi_{i_{1}, l_{1}}-\phi_{i_{2}, l_{2}}\right)}\right) \\
& \times E\left[G_{p}^{B}\left(\Theta_{i_{1} l_{1}}^{B} ; \omega_{1}\right) G_{q}^{B^{*}}\left(\Theta_{i_{2} l_{2}}^{B} ; \omega_{2}\right)\right. \\
& \times e^{\left.j\left(\left(\omega_{1} / c\right) a_{p}^{B^{T}} \Theta_{i_{1} l_{1}}^{B}-\left(\omega_{2} / c\right) a_{q}^{B^{T}} \Theta_{i_{2} l_{2}}^{B}\right)\right]} \\
& \times E\left[G_{m}^{M}\left(\Theta_{i_{1} l_{1}}^{M} ; \omega_{1}\right) G_{n}^{M^{*}}\left(\Theta_{i_{2} l_{2}}^{M} ; \omega_{2}\right)\right. \\
& \left.\left.\times e^{j\left(\left(\omega_{1} / c\right)\left(a_{m}^{M}-V t_{1}\right)^{T} \Theta_{i_{1} l_{1}}^{M}-\left(\omega_{2} / c\right)\left(a_{n}^{M}-V t_{2}\right)^{T} \Theta_{i_{2} l_{2}}^{M}\right)}\right]\right\}
\end{aligned}
$$

Equations $(8) \div(14)$ present the calculation methodology of the expectations in (7). We present the calculation methodology at the MS side but one should note that the same calculation procedure is valid for the BS side. The calculation of this equation is performed for two cases.

Case 1. The paths are considered similar and this is equivalent to $i_{1}=i_{2}=i, l_{1}=l_{2}=l$; some of the random variables in this expression become identical and, by using the Fourier series 
expansions for both APPs and AASs, we are able to calculate these expectations either at the BS or at the MS.

Case 2. The paths are considered to be dissimilar. This is equivalent to $i_{1} \neq i_{2}, l_{1} \neq l_{2}$; we assume that the DOD or DOA PDFs of different propagation waves are independent of each other; that is, $\Theta_{i_{1} l_{1}}^{M}$ and $\Theta_{i_{2} l_{2}}^{M}$ (or $\Theta_{i_{1} l_{1}}^{B}$ and $\Theta_{i_{2} l_{2}}^{B}$ ) are independent.

(i) The calculation of the first expectation in (7) is carried out as follows:

$$
\begin{aligned}
& E\left[\left(\tau_{i_{2} l_{2}} \tau_{i_{1} l_{1}}\right)^{-1} e^{j\left(\omega_{2} \tau_{i_{2} l_{2}}-\omega_{1} \tau_{i_{1} l_{1}}\right)}\right. \\
& \left.\times e^{\left(\omega_{1} T_{m, p, l_{1}}-\omega_{2} T_{m, p, l_{2}}\right)}\right]
\end{aligned}
$$

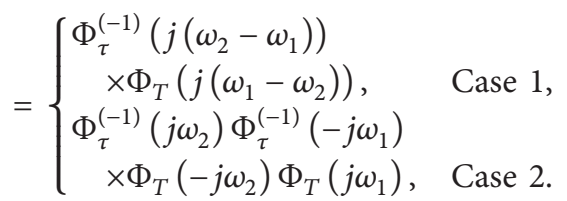

The elements denoted by $\Phi_{\tau, T}(s)$ represent the moment generating functions (MGFs) of the delay profile $(\tau, T)$ evaluated at the difference between two frequencies. $\Phi_{\tau, T}(s)$ suggests that channel similarity depends on frequency differences and not on absolute frequencies. The value of this parameter tends to decay when the frequency differences increase. The definition of the MGF of a random variable $x$ with the PDF $f_{X}(x)$ is defined as follows [30, page 415]: $\Phi_{X}(s)=E\left[e^{j s X}\right]=$ $\int_{-\infty}^{\infty} e^{j s \xi} f_{X}(\xi) d \xi$. The probability densities of the absolute times of arrival of clusters and paths used to calculate the MGFs are represented by [31]

$$
p_{T_{l}}(T)=\frac{\Lambda^{L+1} T^{L} e^{-\Lambda T}}{L !}, \quad p_{\tau_{i, l}}(\tau)=\frac{\lambda^{I} \tau^{I-1} e^{-\lambda \tau}}{(I-1) !}
$$

where $\Lambda$ and $\lambda$ are the cluster arrival rate and ray arrival rate. The parameter $1 / \Lambda$ is typically in the range of $10-50 \mathrm{~ns}$, while $1 / \lambda$ shows wide variations from $0.5 \mathrm{~ns}$ in non-line-of-sight (NLOS) situations to more than $5 \mathrm{~ns}$ in LOS situations [32].

(ii) The calculation of the second expectation in (7) is performed as follows.

Considering the case of the planar wave propagation, we take into account the phase contribution of surrounding scatterers by a random phase change parameter $\phi_{i l} \sim U[-\pi, \pi)$. Since the path phase shifts, $\phi_{i l}$, appear in (4) in the form of $e^{j \phi_{i l}}$, the correlations of $e^{j \phi_{i l}}$ over different paths and clusters, $E\left[e^{j\left(\phi_{i_{1} l_{1}}-\phi_{i_{2} l_{2}}\right)}\right]$, have impact on the channel characteristics. We assume that $E\left[e^{j\left(\phi_{i_{1} l_{1}}-\phi_{i_{2} l_{2}}\right)}\right]$ can take the following values:

$$
E\left[e^{j\left(\phi_{i_{1}, l_{1}}-\phi_{i_{2}, l_{2}}\right)}\right]= \begin{cases}1, & \text { Case 1, } \\ k^{2}, & \text { Case 2. }\end{cases}
$$

(iii) The calculation of the third expectation in (7) is based on the following methodology:

Case $1: E\left[G_{m}^{M}\left(\Theta_{i l}^{M} ; \omega_{1}\right) G_{n}^{M^{*}}\left(\Theta_{i l}^{M} ; \omega_{2}\right)\right.$

$$
\begin{gathered}
\left.\times e^{j\left(\left(\omega_{1} / c\right)\left(a_{m}^{M}-V t_{1}\right)^{T} \Theta_{i l}^{M}-\left(\omega_{2} / c\right)\left(a_{n}^{M}-V t_{2}\right)^{T} \Theta_{i l}^{M}\right)}\right] \\
=\int_{-\pi}^{\pi} G_{m}^{M}\left(\Theta_{i l}^{M} ; \omega_{1}\right) G_{n}^{M^{*}}\left(\Theta_{i l}^{M} ; \omega_{2}\right) \\
\times e^{(j / c)\left(d_{m n}^{M}\right)^{T} \Theta_{i l}^{M}} f_{\Theta^{M}}\left(\Theta_{i l}^{M}\right) d \Theta_{i l}^{M} \\
=\int_{-\pi}^{\pi} \sum_{k=-\infty}^{\infty}\left(\mathscr{G}_{m, k}^{M}\left(\omega_{1}\right) \otimes \mathscr{G}_{n,-k}^{M^{*}}\left(\omega_{2}\right) \otimes \mathscr{F}_{k}^{M}\right) \\
\quad \times e^{j k \Theta_{i l}^{M}+j\left(\left|d_{m n}^{M}\right| \cos \left(\Theta_{i l}^{M}-\angle d_{m, n}^{M}\right) / c\right)} d \Theta_{i l}^{M} \\
=2 \pi \sum_{k=\infty}^{\infty} j^{k} e^{j k \angle d_{m, n}^{M}}
\end{gathered}
$$

$$
\times\left(\mathscr{G}_{m, k}^{M}\left(\omega_{1}\right) \otimes \mathscr{G}_{n,-k}^{M^{*}}\left(\omega_{2}\right) \otimes \mathscr{F}_{k}^{M}\right) J_{k}\left(\frac{\left|d_{m n}^{M}\right|}{c}\right),
$$

where $\mathscr{G}_{m, k}^{M}, \mathscr{G}_{n, k}^{M}$, and $\mathscr{F}_{k}^{M}$ are the FSCs of the APPs (defined in (1)) and the AAS (defined in (2)) in the corresponding coordinates, respectively, $J_{k}(u) \triangleq\left(j^{-k} / \pi\right) \int_{0}^{\pi} e^{j(k \xi+u \cos \xi)} d \xi$ is the $k$ th-order Bessel function, $\otimes$ is the linear convolution, and $|\cdot|$ is the Euclidean norm.

The definition of the linear convolution can be formulated as follows [33, page 155]: given two discrete-time signals, $x_{n}$ and $y_{n}$, the linear convolution between them is defined as follows: $z_{n} \triangleq x_{n} \otimes y_{n}=\sum_{k=\infty}^{\infty} x_{k} y_{n-k}$.

The vectors and $d_{m, n}^{M}$ and $d_{p, q}^{B}$ are the separation vectors between two antenna elements at the MS $(m, n)$ and at the BS $(p, q)$. Large distances often result in less STF correlation as the Bessel functions asymptotically decrease. The norms of $d_{m, n}^{M}$ and $d_{p, q}^{B}$ represent shifted distances (between $\omega_{1} a_{p}^{B}$ and $\left.\omega_{2} a_{q}^{B}\right)$ at the BS and (between $\omega_{2}\left(t_{2} V+a_{n}^{M}\right)$ and $\omega_{1}\left(t_{1} V+\right.$ $\left.\left.a_{m}^{M}\right)\right)$ at the MS. Parameters $d_{(\cdot, \cdot)}^{(\cdot)}$ contain space, time, and frequency separations between $h_{p m}\left(t_{1}, \omega_{1}\right)$ and $h_{q n}\left(t_{2}, \omega_{2}\right)$. These two vectors, illustrate theimpact of the antennas' location (spatial correlation), of thecarrier frequencies (frequency correlation), of the time indices and of the mobile speed on the CCF at BS and MS. The value of the $d_{m, n}^{M}$ and $d_{p, q}^{B}$ can be determined based on the following equations:

$$
\begin{gathered}
d_{m}^{M} \triangleq \omega_{1}\left(a_{m}^{M}-t_{1} V\right), \quad d_{n}^{M} \triangleq \omega_{2}\left(a_{n}^{M}-t_{2} V\right), \\
d_{m, n}^{M} \triangleq\left(\omega_{2} t_{2}-\omega_{1} t_{1}\right) V+\left(\omega_{1} a_{m}^{M}-\omega_{2} a_{n}^{M}\right), \\
d_{p}^{B} \triangleq \omega_{1} d_{p}^{B}, \quad d_{q}^{B} \triangleq \omega_{2} d_{q}^{B}, \quad d_{p, q}^{B} \triangleq \omega_{1} a_{p}^{B}-\omega_{2} a_{q}^{B} .
\end{gathered}
$$


Similar to the calculation in the first case, for the second case, we have (in MS)

$$
\begin{aligned}
\text { Case } 2: E[ & G_{m}^{M}\left(\Theta_{i_{1} l_{1}}^{M} ; \omega_{1}\right) G_{n}^{M^{*}}\left(\Theta_{i_{2} l_{2}}^{M} ; \omega_{2}\right) \\
& \left.\times e^{j\left(\left(\omega_{1} / c\right)\left(a_{m}^{M}-V t_{1}\right)^{T} \Theta_{i_{1} l_{1}}^{M}-\left(\omega_{2} / c\right)\left(a_{n}^{M}-V t_{2}\right)^{T} \Theta_{i_{2}, l_{2}}^{M}\right)}\right] \\
= & \left(2 \pi^{2}\right) \\
\times & {\left[\sum_{k=-\infty}^{\infty} j^{k} e^{j k\left\langle d_{m}^{M}\right.}\left(\mathscr{G}_{m, k}^{M}\left(\omega_{1}\right) \otimes \mathscr{F}_{k}^{M}\right) J_{k}\left(\frac{\left|d_{m}^{M}\right|}{c}\right)\right] } \\
\times & {\left[\sum_{k=-\infty}^{\infty} j^{k} e^{j k \angle d_{n}^{M}}\left(\mathscr{G}_{n,-k}^{M}\left(\omega_{2}\right) \otimes \mathscr{F}_{k}^{M}\right) J_{k}\left(\frac{\left|d_{n}^{M}\right|}{c}\right)\right] . }
\end{aligned}
$$

We formulate the CCF including both cases using the derivations presented above:

$$
\begin{aligned}
R_{p m, q n}\left(t_{1}, t_{2}, \omega_{1}, \omega_{2}\right) \\
=\frac{\left(\omega_{b w 1} \omega_{b w 2}\right)^{\eta}}{\left(\omega_{1} \omega_{2}\right)^{\eta}\left(4 \omega_{1} \omega_{2}\right)} \\
\times \Phi_{\tau}^{-(1)}\left(j\left(\omega_{1}-\omega_{2}\right)\right) \Phi_{T}\left(j\left(\omega_{2}-\omega_{1}\right)\right) \\
\quad \times\left\{\mathscr{W}\left(d_{p, q}^{B}, \mathscr{H}_{k}^{B}\right) \times \mathscr{W}\left(d_{m, n}^{M}, \mathscr{H}_{k}^{M}\right)\right\} \\
+k^{2} \Phi_{\tau}^{(-1)}\left(j \omega_{2}\right) \Phi_{\tau}^{(-1)}\left(-j \omega_{1}\right) \Phi_{T}\left(-j \omega_{2}\right) \Phi_{T}\left(j \omega_{1}\right) \\
\times\left\{\mathscr{W}\left(d_{p}^{B}, \mathscr{G}_{p, k}^{B}\left(\omega_{1}\right) \otimes \mathscr{F}_{k}^{B}\right)\right. \\
\quad \times \mathscr{W}\left(d_{q}^{B}, \mathscr{G}_{q,-k}^{B *}\left(\omega_{2}\right) \otimes \mathscr{F}_{k}^{B}\right) \\
\quad \times \mathscr{W}\left(d_{m}^{M}, \mathscr{G}_{m, k}^{M}\left(\omega_{1}\right) \otimes \mathscr{F}_{k}^{M}\right) \\
\left.\quad \times \mathscr{W}\left(d_{n}^{M}, \mathscr{G}_{n,-k}^{M *}\left(\omega_{2}\right) \otimes \mathscr{F}_{k}^{M}\right)\right\} .
\end{aligned}
$$

We evaluate the CCF presented in (14) for the scenario, $i_{1}=$ $i_{2}=i, l_{1}=l_{2}=l$, and $k^{2}=0$. The final expression of the CCF obtained in this scenario is presented in

$$
\begin{aligned}
& R_{p m, q n}\left(t_{1}, t_{2}, \omega_{1}, \omega_{2}\right) \\
&= \frac{\left(\omega_{b w 1} \omega_{b w 2}\right)^{\eta}}{\left(\omega_{1} \omega_{2}\right)^{\eta}\left(4 \omega_{1} \omega_{2}\right)} \\
& \quad \times \Phi_{\tau}^{-(1)}\left(j\left(\omega_{2}-\omega_{1}\right)\right) \Phi_{T}\left(j\left(\omega_{2}-\omega_{1}\right)\right) \\
& \quad \times \mathscr{W}\left(d_{p q}^{B}, \mathscr{G}_{p, k}^{B}\left(\omega_{1}\right) \otimes \mathscr{G}_{q,-k}^{B *}\left(\omega_{2}\right) \otimes \mathscr{F}_{k}^{B}\right) \\
& \quad \times \mathscr{W}\left(d_{m n}^{M}, \mathscr{G}_{m, k}^{M}\left(\omega_{1}\right) \otimes \mathscr{G}_{n,-k}^{M *}\left(\omega_{2}\right) \otimes \mathscr{F}_{k}^{M}\right),
\end{aligned}
$$

where

$$
\begin{array}{r}
\mathscr{W}\left(d, \mathscr{H}_{k}\right) \triangleq 2 \pi \sum_{k=-\infty}^{\infty} j^{k} e^{j k L d} \mathscr{H}_{k}(\omega) J_{k}\left(\frac{|d|}{c}\right), \\
\mathscr{H}_{k}=\mathscr{G}_{k}\left(\omega_{1}\right) \otimes \mathscr{G}_{-k}^{*}\left(\omega_{2}\right) \otimes \mathscr{F}_{k} .
\end{array}
$$

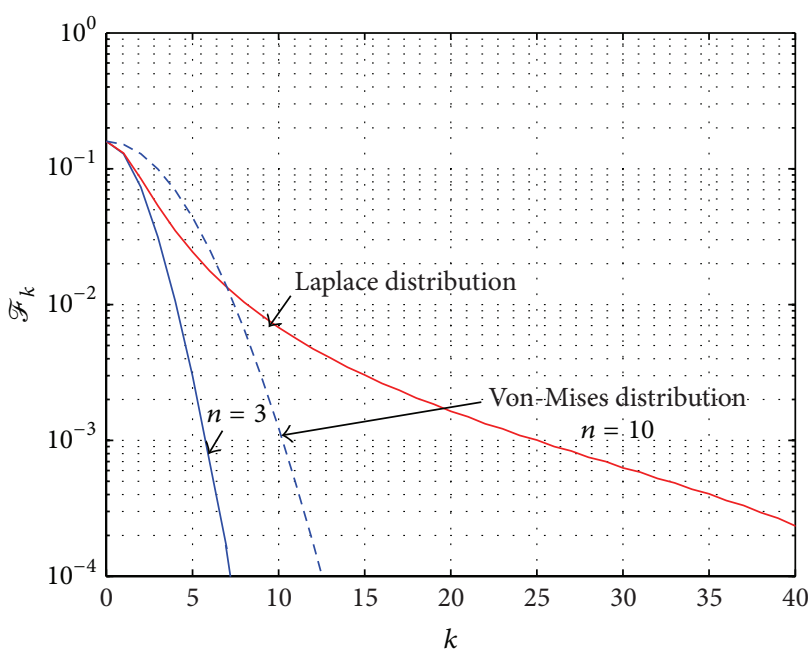

FIGURE 1: Fourier series coefficients for two AAS PDFs, to approximate Laplace and von-Mises distributions (with orders of nonisotropy $n=3,10$ ) determined to correspond to the real PDFs for the nonisotropic propagation environment.

The CCF appeared to be the convolution of two categories of FSCs as follows.

(i) $\mathscr{F}_{k}^{(\cdot)}$ are the $k$ th FSCs of PDFs of the nonisotropic AAS at both MS and BS sides. This means that the $\mathrm{CCF}$ is represented as the same linear combination of FSCs associated with these PDFs. This allows accurate modeling for various $2 \mathrm{D}$ wireless propagation environments. In our model, we determined the necessary number of FSCs by calculating integral (2) for each of the two PDFs that describe the nonisotropic propagation environment. In Figure 1 the FSCs of Laplace and von-Mises PDFs (at the MS) are compared. For the von-Mises distribution, FSCs are presented when the propagation environment has two different orders of nonisotropy.

(ii) $\mathscr{G}_{(\cdot, k)}^{(\cdot)}$ are the $k$ th FSCs of $2 \mathrm{D} \mathrm{WB} / \mathrm{UWB}$ APPs, at both $\mathrm{BS}$ and MS sides, used to investigate the impact of directional and omnidirectional antennas. At this point we differentiate the WB APP which is not frequency selective from the frequency selective UWB APPs.

3.1. CCF Numerical Evaluation. Low correlation between received signals is a necessary condition for good MIMO performances. This low correlation is achieved when each antenna provides a unique weighting for each $l$ cluster with I multipath components. In order to achieve space diversity with MIMO systems, the antennas need to be as compact as possible but also to be able to recover signals with dissimilar multipath fading. An optimal solution may be a low threshold for correlation rather than zero correlation between antennas. Low correlation is often considered when CCF $<0.7$ [25]. Correlation values are especially significant in applications where the evaluation of the channel capacity is necessary. 


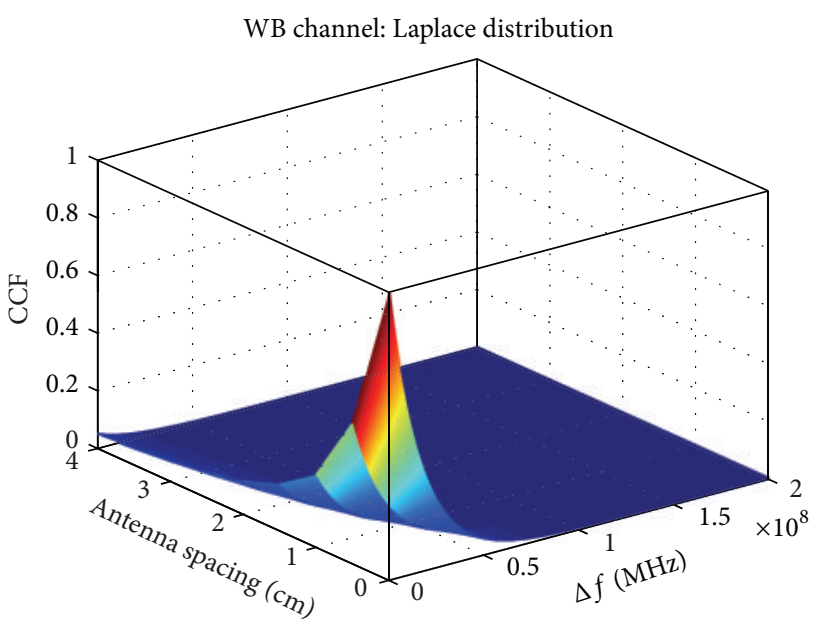

UWB channel: Laplace distribution

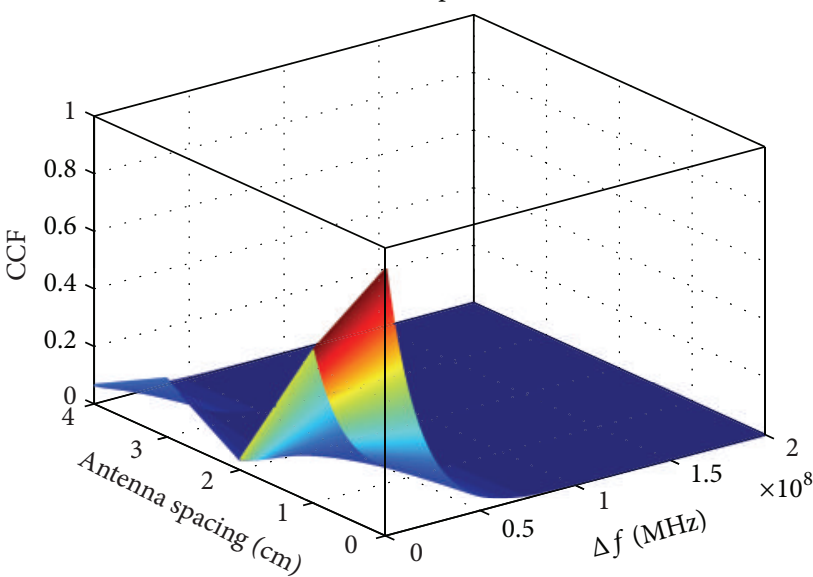

WB channel: von-Mises distribution

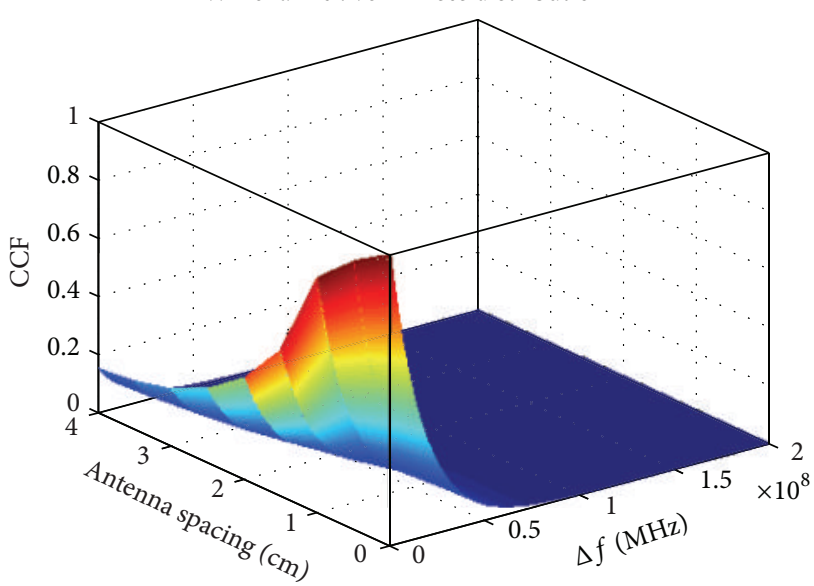

UWB channel: von-Mises distribution

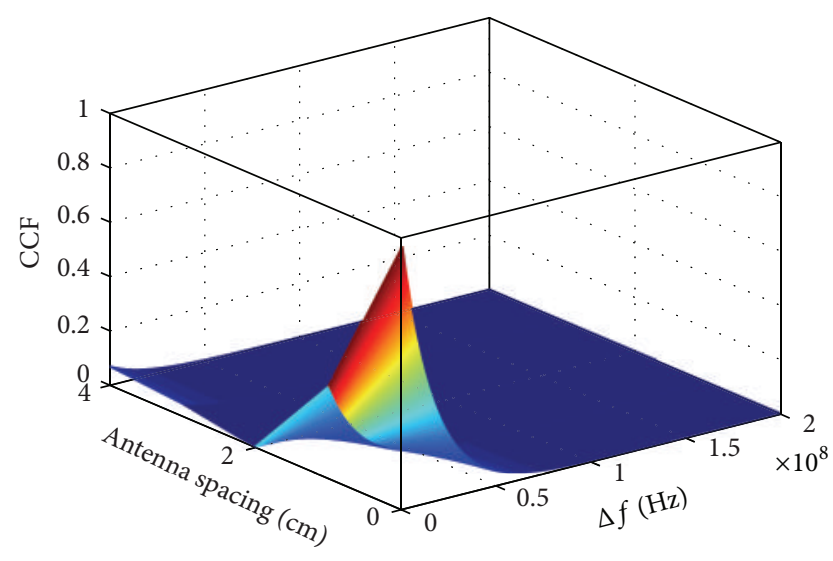

FIgURE 2: CCF corresponding to WB and UWB signals with omnidirectional APP, Laplace, and von-Mises PDFs of the propagation environment, with antenna spacing $\lambda / 2=c \pi / \omega_{H}$ and frequency offsets $\Delta f=f_{2}-f_{1}$.

Figures 2 and 3 depict the effect of the distribution of the nonisotropic propagation environment, the APP (directional, omnidirectional), the antenna spacing, and the carrier frequency offset, $\Delta f=f_{2}-f_{1}$ (where $f_{1}$ is constant at $5 \mathrm{GHz}$ for WB channels and at $3.1 \mathrm{GHz}$ for UWB channels) on the normalized CCF $\left|R\left(t_{1}, t_{2}, \omega_{1}, \omega_{2}\right)\right|^{2} /\left|R\left(t_{1}, t_{1}, \omega_{1}, \omega_{1}\right)\right|^{2}$. In the reported figures, the unit for the antenna spacing is half of the highest frequency of the signal bandwidth, $\lambda / 2=c \pi / \omega_{H}$, where $\omega_{H}=2 \pi f_{H}$.

In [22, page 26] measurement results for spatial correlation of UWB MIMO channels with omnidirectional antennas are presented. The measured data show that the spatial correlation is a monotonously decreasing function of the antenna distance. A complete characterization of MIMO UWB channels correlations is provided in [34]. It was stated that, for about $4 \mathrm{~cm}$ antenna spacing, the correlation function follows a pattern of the first kind zeroth-order Bessel function with distance. It was also found that a value of $2 \mathrm{~cm}$ for antenna spacing is sufficient for CCF $\leq 0.5$. In [35-37] it was stated that the CCF values are higher for directional antennas than for omnidirectional antennas. This is because their beamwidth limits the effective angular spread and their ability to capture multipath signals from all directions. The results, regarding the spatial correlation, presented in Figures 2 and 3 , are consistent with the CCF's behavior described in the mentioned references. In the reported figures the influence of the frequency correlation is also evaluated. It can be observed how CCF decreases as $\Delta f$ increases. This decrease results not only from the Bessel functions (as the central frequencies appear in the Bessel operands) but also from the term produced by the MGF of the delay profile. As the figures show, the CCF is maximum when $\Delta f=0$ and when there is no separation between the antennas of the MIMO system. CCF also decreases when WB APP is replaced by UWB APP. This is because UWB signals offer a higher degree of diversity owing to their abundant multipaths. In Figure 1 it can be observed that the number of FSCs necessary to approximate Laplace PDF is larger than the number of FSCs necessary to approximate von-Mises distribution. This indicates that a propagation environment described by Laplace PDF is characterized by a higher order of nonisotropy which provides better diversity and lower correlation between MIMO subchannels. The graphical representations of CCF show that the correlation decreases faster when the environment has 


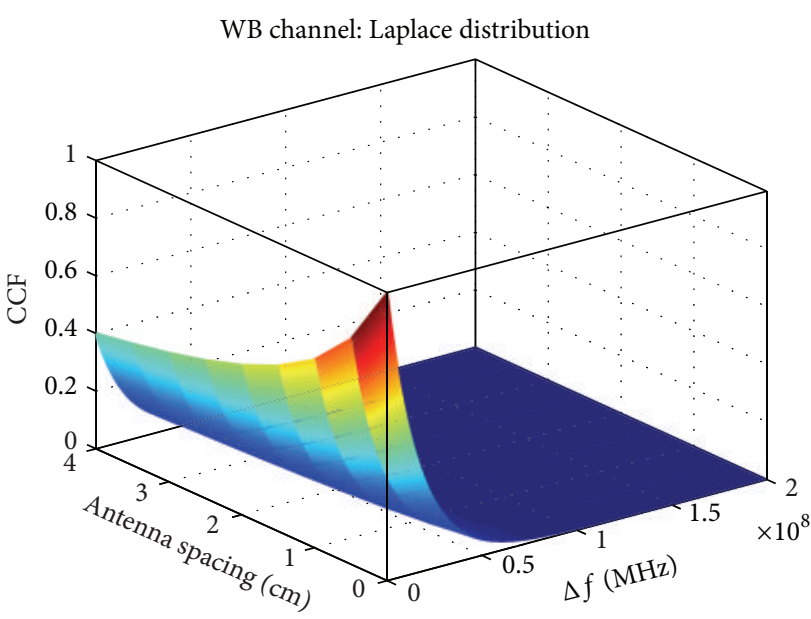

UWB channel: Laplace distribution

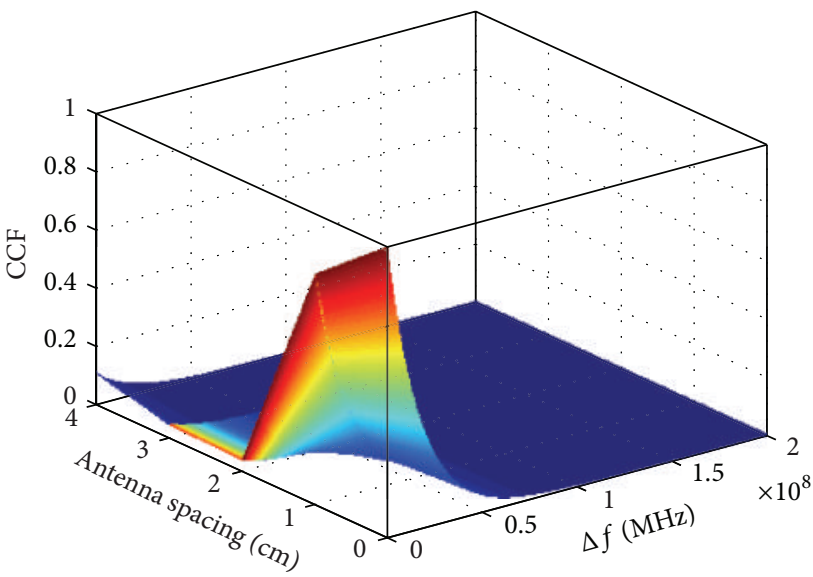

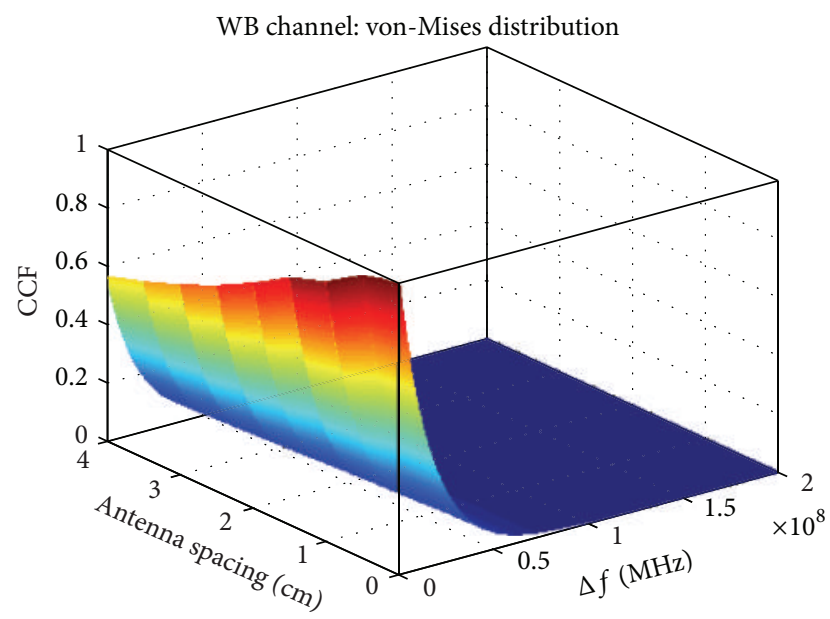

UWB channel: von-Mises distribution

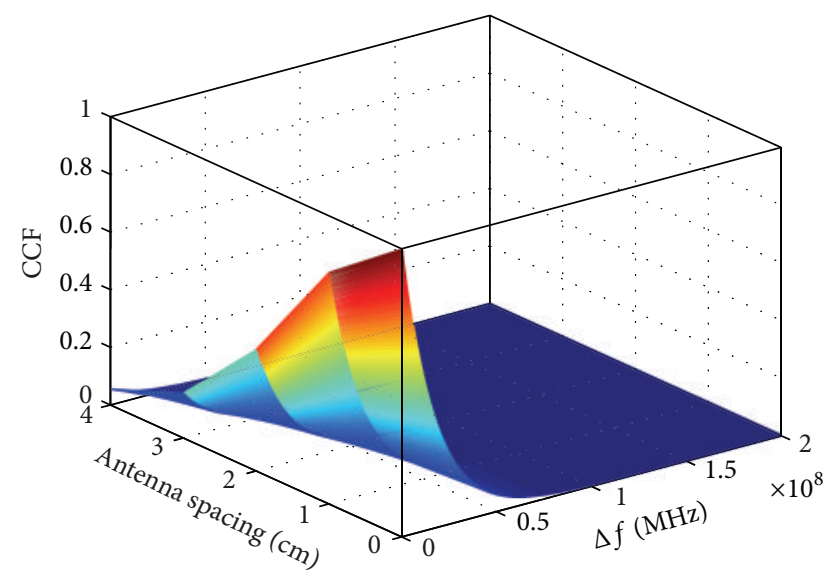

FIGURE 3: CCF corresponding to WB and UWB signals with directional APP, Laplace, and von-Mises PDFs of the propagation environment, with antenna spacing $\lambda / 2=c \pi / \omega_{H}$ and frequency offsets $\Delta f=f_{2}-f_{1}$.

Laplacian distribution, for both WB and UWB channels. In [38] the influence of parameters like antenna element spacings, environment parameters, and scattering density, on the spatial correlation properties of multilink MIMO channels, was presented. Based on different propagation phenomena like those previously mentioned new key technical issues in developing and realizing multiinput multioutput technology were presented in [39].

\section{Fourier Analysis of the 2D STF-CCF of WB/UWB MIMO Channels}

The derived CCF can be used to analyze the power spectral density (PSD) of WB and UWB MIMO channels. PSD gives the distribution of the signal power among various frequencies and also reveals the presence or the absence of repetitive patterns and correlation sequences in the signal process. These structural patterns are useful in a wide range of applications like data forecasting, signal detection and coding, pattern recognition, and radar and decision-making systems. The analyzed PSD corresponds to a multiple-input single-output (MISO) channel which is a particular case of MIMO channel. This analysis corresponds to the stationary scenario when $\omega_{1}=\omega_{2}=\omega$ and $m=n=1$. Considering $\angle d_{1,1}^{M}=\angle V+\angle\left(t_{2}-t_{1}\right)$ we get

$$
\begin{aligned}
& R_{p 1, q 1}\left(t_{1}, t_{2}, \omega, \omega\right) \\
& =\pi \frac{\omega_{b w}^{2 \eta}}{2 \omega^{2 \eta+2}} \mathscr{W}\left(d_{p, q}^{B}, \mathscr{H}_{k}^{B}\right) \\
& \times \sum_{k=-\infty}^{\infty} j^{k} e^{j k \Delta V}\left(\mathscr{G}_{1, k}^{M}(\omega) \otimes \mathscr{G}_{1,-k}^{M *}(\omega) \otimes \mathscr{F}_{k}^{M}\right) \\
& \quad \times J_{k}\left(\frac{\omega\left(t_{2}-t_{1}\right)|V|}{c}\right) .
\end{aligned}
$$

Using the Fourier transform of $J_{k}(u)$, which is given by

$$
\begin{aligned}
J_{k}(\Lambda) \triangleq F\left[J_{k}(u)\right] & =\int_{-\infty}^{\infty} e^{-j \Lambda \xi} J_{k}(\xi) d \xi \\
& = \begin{cases}\left(2(-j)^{k} T_{k}(\Lambda)\right) / \sqrt{1-\Lambda^{2}}, & |\Lambda|<1, \\
0, & |\Lambda| \geqq 1,\end{cases}
\end{aligned}
$$

where $F[\cdot]$ denotes the Fourier transform and $T_{k}(\Lambda) \triangleq$ $\cos \left[k \cos ^{-1}(\Lambda)\right]$ is the $k$ th-order Chebyshev polynomial 


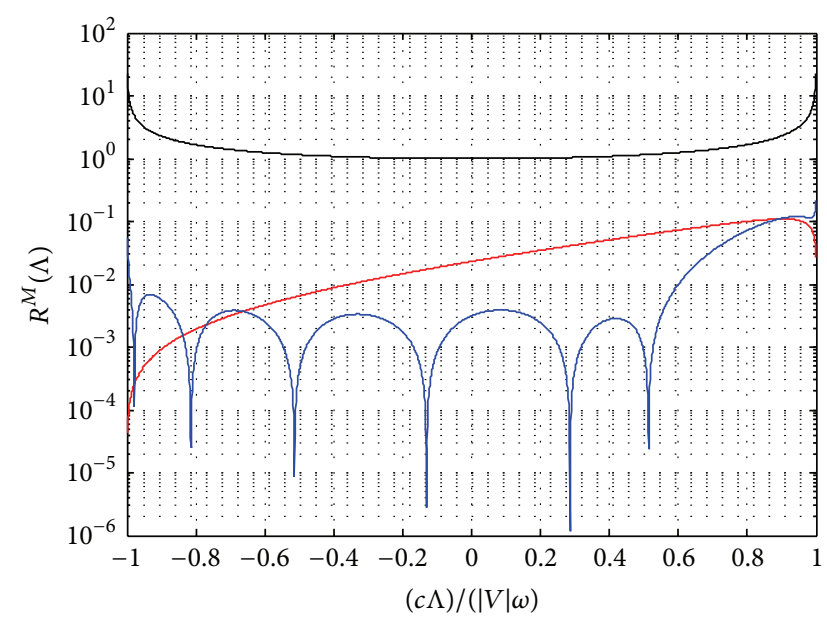

(a) Helical antenna

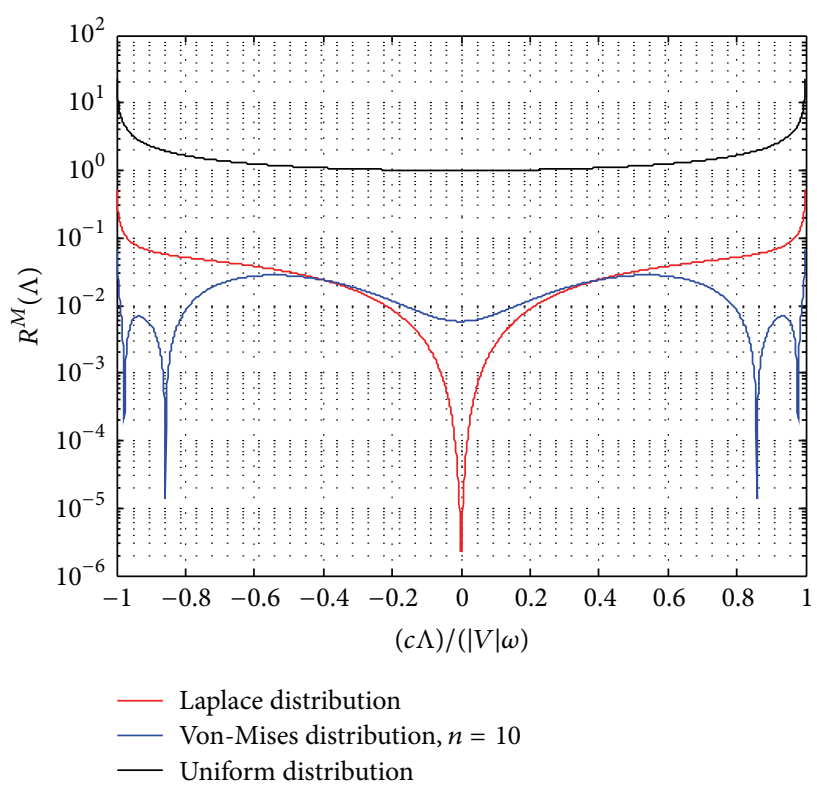

(c) Helical antenna

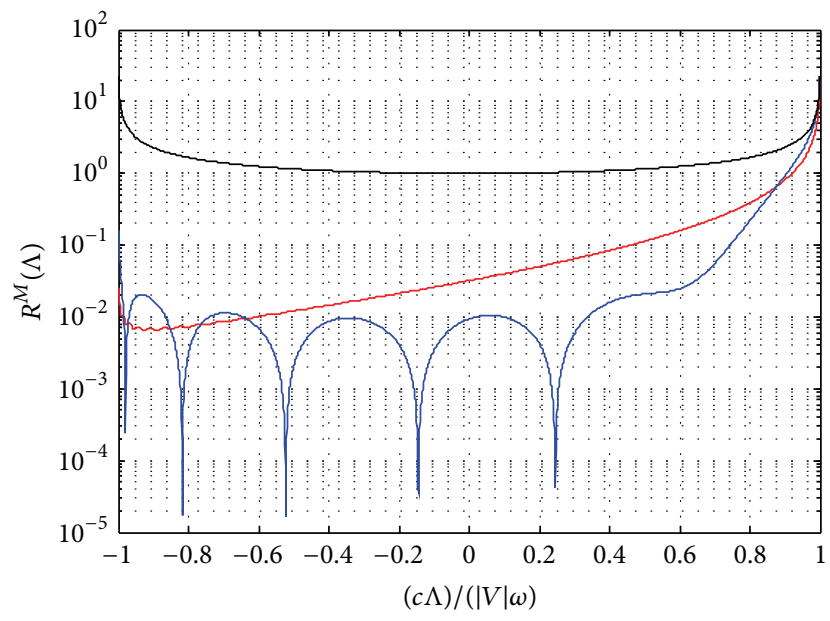

(b) Rectangular antenna

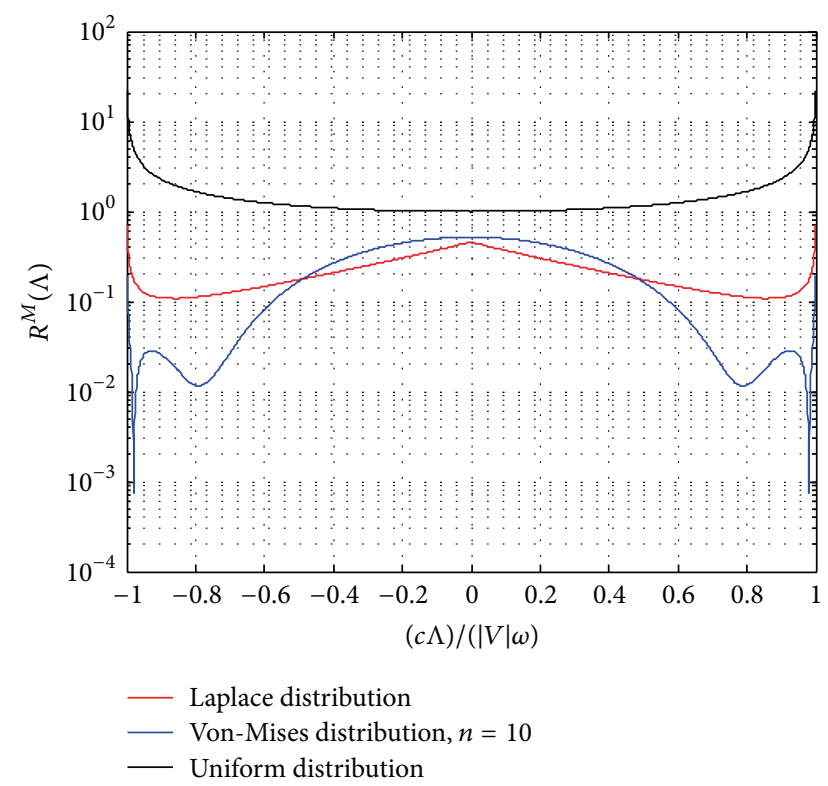

(d) Rectangular antenna

FIGURE 4: PSD of WB channels, MS moves in the positive direction of the $x$-axis ((a), (b)) and $y$-axis ((c), (d)), two antenna types employed at the MS, nonisotropic propagation (Laplacian or von-Mises distributed), and isotropic environment (uniformly distributed).

function of the first kind [[16], page 486], the Fourier transform of the CCF derived for stationary case versus the time-difference index, $\Delta t \triangleq t_{2}-t_{1}$, results in the following equation:

$$
\begin{aligned}
R_{p 1, q 1} & (\Lambda, \omega) \\
\triangleq & \int_{-\infty}^{\infty} e^{-j \Lambda \Delta t} R_{1 p, 1 q}\left(t_{1}, t_{2}, \omega, \omega\right) d \Delta t \\
= & \frac{\omega_{b w}^{2 \eta}}{2 \omega^{2 \eta+2}} \mathscr{W}\left(d_{q, p}^{M}, \mathscr{H}_{k}^{B}\right) \frac{\pi c}{|V|} \\
& \times \sum_{k=-\infty}^{\infty}\left[\left(e^{j k\langle V} \mathscr{G}_{1, k}^{M}(\omega) \otimes \mathscr{G}_{1,-k}^{M *}(\omega) \otimes \mathscr{F}_{k}^{M}\right) C_{k}\right],
\end{aligned}
$$

where $\mathscr{H}_{k}^{B} \triangleq \mathscr{G}_{p, k}^{B}(\omega) \otimes \mathscr{G}_{q,-k}^{B *}(\omega) \otimes \mathscr{F}_{k}^{B}$ and $\Lambda$ is a frequency variable in the interval $-(\omega / c)|V|<\Lambda<(\omega / c)|V|$. Note that $R_{p 1, q 1}(\Lambda, \omega)=0$ for all $|\Lambda| \geqslant(\omega / c)|V|$. We denote $C_{k}=\left(T_{k}(c \Lambda /|V| \omega)\right) / \sqrt{1-(c \Lambda /|V| \omega)^{2}} ; T_{k}(\cdot)$ is the Chebyshev polynomials which form a complete orthogonal set on the interval $-1 \leqslant u<1$, with respect to the weighting function $1 / \sqrt{1-u^{2}}$.

Therefore, any bandlimited CCF (in the interval $-(\omega / c)|V| \leqslant \Lambda \leqslant(\omega / c)|V|)$ can be expanded in terms of Chebyshev polynomials as shown in (19). The PSD, $R^{M}(\Lambda)$, is the last term in (19) and shows the channel variations caused around or by the MS speed and direction and the impact of 


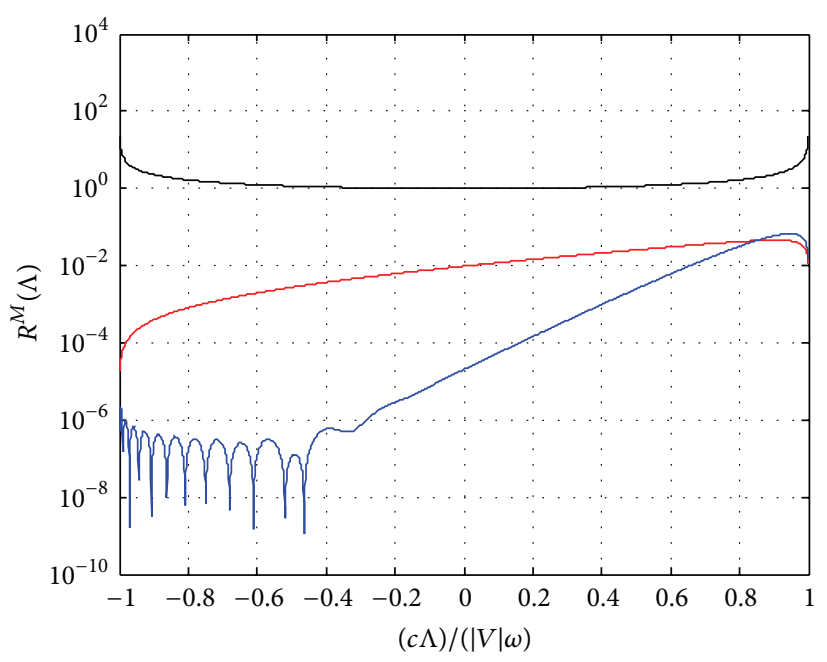

(a) Helical antenna

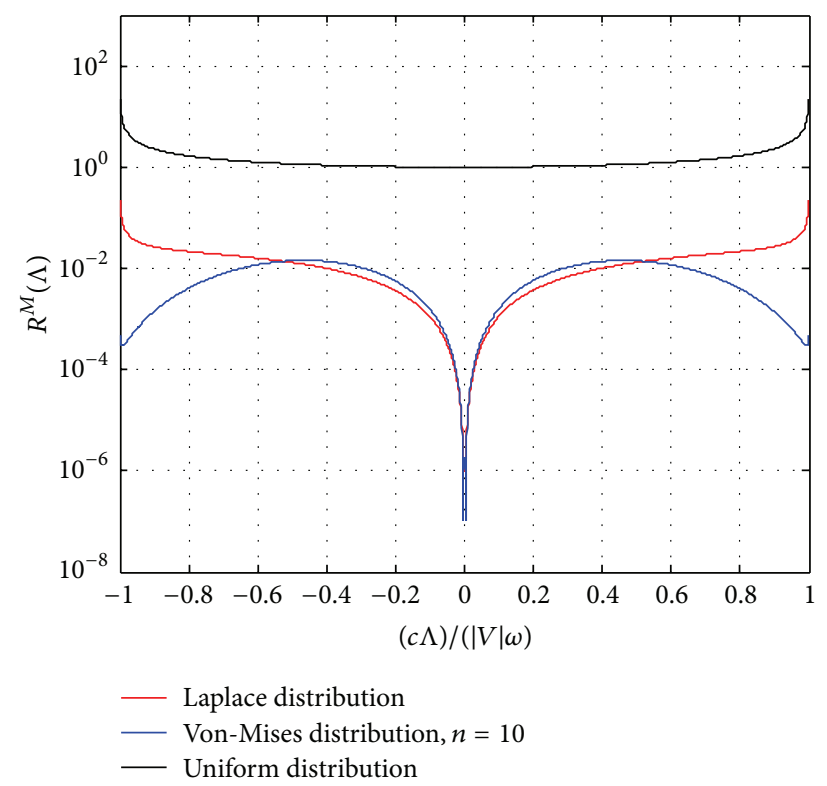

(c) Helical antenna

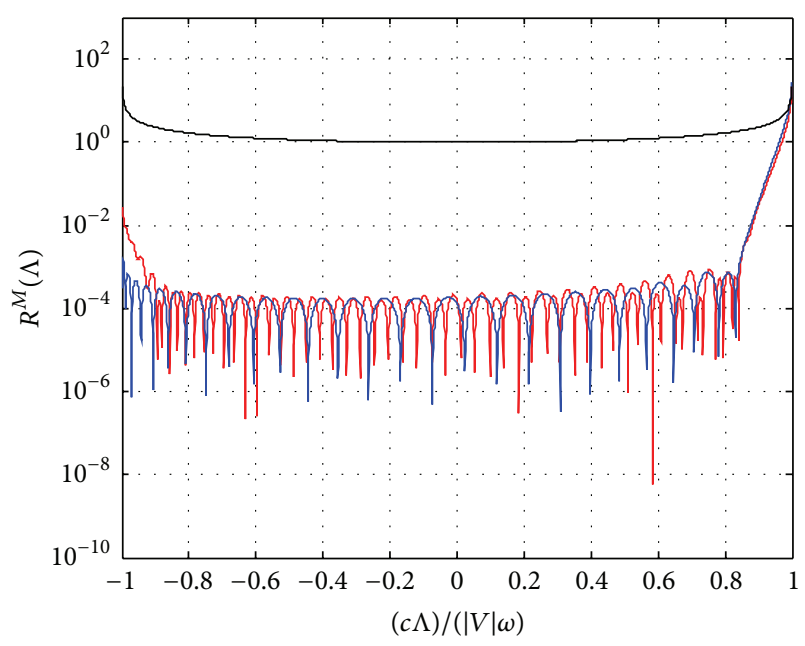

(b) Rectangular antenna

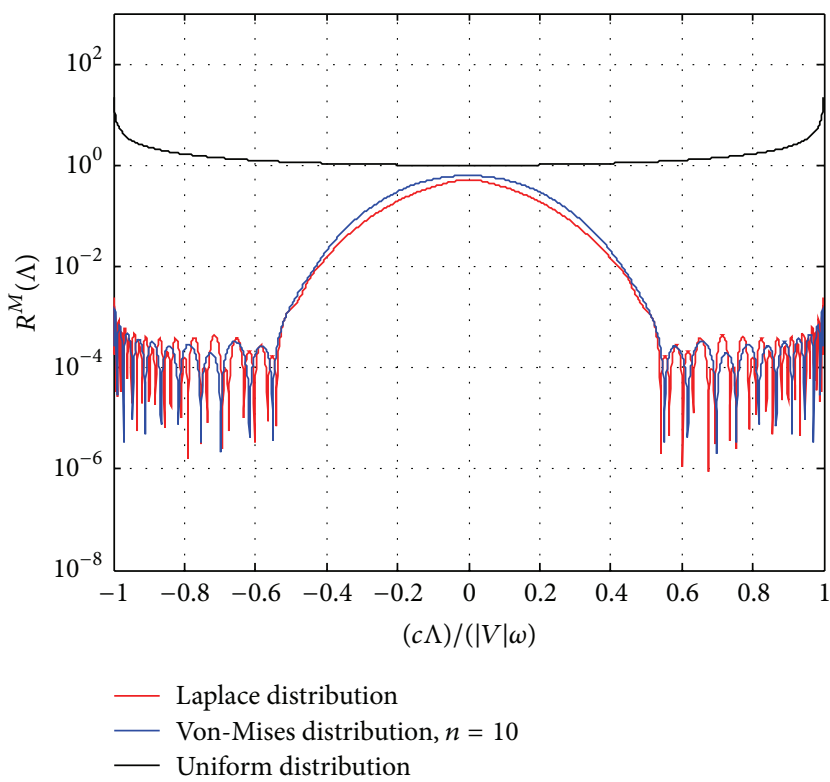

(d) Rectangular antenna

FIGURE 5: PSD of UWB channels, MS moves in the positive direction of the $x$-axis ((a), (b)) and $y$-axis ((c), (d)), two antenna types employed at the MS, nonisotropic propagation (Laplacian or von-Mises distributed), and isotropic environment (uniformly distributed).

the nonisotropic environment, of the channel bandwidth, and of the APP:

$$
R^{M}(\Lambda) \triangleq \sum_{k=-\infty}^{\infty} e^{j k L V}\left(\mathscr{G}_{1, k}^{M}(\omega) \otimes \mathscr{G}_{1,-k}^{M *}(\omega) \otimes \mathscr{F}_{k}^{M}\right) C_{k}
$$

In Figures 4 and 5, the PSD of WB and UWB signals is depicted. The presented results illustrate (20) with the influence of the following elements.

(i) The channel bandwidth for WB channels was equal to $200 \mathrm{MHz}$ with the central frequency $f=2.5 \mathrm{GHz}$. For UWB channels the results correspond to a bandwidth delimited by $3.1 \div 10.6 \mathrm{GHz}$, (ii) APPs, specific to WB and UWB systems, employed at the MS side (directional and omnidirectional antennas),

(iii) nonisotropic propagation environment around the MS (Laplace and von-Mises PDFs),

(iv) direction of the MS speed, the positive $x$-axis or $y$ axis direction.

From the comparative analysis of PSD obtained for $\mathrm{WB}$ and UWB channels relevant conclusions can be drawn regarding its distribution in the interval $-(\omega / c)|V|<\Lambda<$ $(\omega / c)|V|$ as follows.

(i) The PSD fluctuations are more pronounced in the case of UWB channels compared to WB channels. This is 
the effect of the frequency selectivity phenomenon whose effects increase with signal frequency and bandwidth. The noticeable increase of frequency selectivity that occurs in the case of UWB channels clearly differentiated them from other channels with narrower bandwidth. This characteristic may be helpful in the identification process of the channel's type for its bandwidth from its PSD only. In Figures 4(a), 4(b), 5(a), and 5(b) it can be observed that MS movement in the positive direction of the $x$ axis produces a PSD with larger values at positive $\Lambda$ than at negative $\Lambda$. This asymmetry of the PSD is also determined by the Doppler spectrum which concentrates towards positive frequency axis.

(ii) The PSD shape is asymmetrical (Figures 4(a), 4(b), $5(\mathrm{a})$, and $5(\mathrm{~b})$ ) or symmetrical (Figures 4(c), 4(d), $5(c)$, and $5(\mathrm{~d}))$ as a consequence of the interaction between the beam of the antenna pattern, the direction of the MS speed, and the distribution of the propagation directions around the MS. Figures 4(c), $4(d), 5(c)$, and 5(d) depict the shape of the PSD which is symmetrically distributed around $\Lambda=0$, because the PDF which characterizes the nonisotropic propagation and the APPs are symmetrical around $\Theta^{M}=0$ and are perpendicular towards the speed direction. For both WB and UWB channels, the maximum Doppler shift is $(\omega / c)|V|$ (i.e., $R^{M}(\Lambda)=0$, if $|\Lambda| \geqslant$ $(\omega / c)|V|)$.

The obtained results are consistent with those proposed in $[19,37]$. In [19] the PSD of a narrowband channel in nonisotropic propagation environment is similar to the PSD shape we obtained for WB channels. Between our results and those presented in [38] there are similarities regarding the Ushape of the PSD but there are also differences determined by parameters specific to channels with large bandwidths, like frequency selectivity, higher central frequency, and APPs typically used for this type of systems. Comparing the PSD shape obtained for WB channels with the PSD obtained for UWB channels and even with the PSD obtained for narrowband channels in [19] we can conclude that the channel bandwidth has a great influence on the channel power spectrum. With the increase of signal bandwidth larger variations can be observed over (because of the increased frequency selectivity) the PSD of UWB channels.

\section{Conclusion}

In this paper we proposed an outdoor channel model for WB/UWB MIMO systems, with moving receiver, based on the mathematical expression of a 2D STF-CCF. The impact of channel bandwidth, nonisotropic propagation, and omnidirectional and directional antennas on the CCF was evaluated. The increase of the channel bandwidth (from WB to UWB) generated the necessity to develop two approaches for the APPs calculation. The derived CCF appeared to be a combination of linear series expansion of Bessel functions. The coefficients of the CCF expansion are represented by the convolution of the Fourier coefficients of the APPs and the AASs specific to WB and UWB channels. The Fourier transform of the CCF in a stationary scenario showed that the PSD diverges from the U-shaped function, that is, Clarke/Jake model. This deviation depends on the AAS, the employed antennas, and the MS speed direction. The increase of the channel bandwidth also generated a more frequency selective PSD for UWB channels compared to the PSD of WB channels. This information can be useful for applications of signal detection and recognition since the increased frequency selectivity makes the identification of the channel's type for its bandwidth from its PSD only possible.

\section{Conflict of Interests}

The authors declare that there is no conflict of interests regarding the publication of this paper.

\section{Acknowledgments}

This paper has been financially supported within the Project entitled "Horizon 2020: Doctoral and Postdoctoral StudiesPromoting the National Interest through Excellence, Competitiveness and Responsibility in the Field of Romanian Fundamental and Applied Scientific Research," Contract number POSDRU/159/1.5/S/140106. This project is cofinanced by the European Social Fund through Sectoral Operational Programme for Human Resources Development 2007-2013, "Investing in People!"

\section{References}

[1] Y. Ma and M. Pätzold, "Wideband two-ring MIMO channel models for mobile-to-mobile communications," in Proceedings of 10th International Symposium on Wireless Personal Communications, pp. 380-384, Jaipur, India, December 2007.

[2] K. Witrisal and M. Pausini, "Statistical analysis of UWB channel correlation functions," IEEE Transactions on Vehicular Technology, vol. 57, no. 3, pp. 1359-1373, 2008.

[3] N. Gvozdenovic and M. Eric, "Localization of users in multiuser MB OFDM UWB systems based on TDOA principle," in Proceedings of the 19th Telecommunications Forum (TELFOR '11), pp. 326-329, Belgrad, Serbia, November 2011.

[4] Q. Zou, A. Tarighat, and A. H. Sayed, "Performance analysis of multiband-OFDM UWB communications with application to range improvement," IEEE Transactions on Vehicular Technology, vol. 56, no. 6, pp. 3864-3878, 2007.

[5] A. Abdi and M. Kaveh, "A space-time correlation model for multielement antenna systems in mobile fading channels," IEEE Journal on Selected Areas in Communications, vol. 20, no. 3, pp. 550-560, 2002.

[6] T. Santos, J. Karedal, P. Almers, F. Tufvesson, and A. F. Molisch, "Modeling the ultra-wideband outdoor channel: measurements and parameter extraction method," IEEE Transactions on Wireless Communications, vol. 9, no. 1, pp. 282-290, 2010.

[7] M. Di Renzo, F. Graziosi, R. Minutolo, M. Montanari, and F. Santucci, "The ultra-wide bandwidth outdoor channel: from measurement campaign to statistical modelling," Mobile Networks and Applications, vol. 11, no. 4, pp. 451-467, 2006.

[8] C. F. Souza and J. C. R. Dal Bello, "UWB signals transmission in outdoor environments for emergency communications," 
in Proceedings of the 11th IEEE International Conference on Computational Science and Engineering Workshops, pp. 343348, San-Paolo, Italy, July 2008.

[9] Y. Wang, X. Yu, Y. Zhang et al., "Using wavelet entropy to distinguish between humans and dogs detected by UWB radar," Progress in Electromagnetics Research, vol. 139, pp. 335-352, 2013.

[10] W. Zhou, J. T. Wang, H. W. Chen, and X. Li, "Signal model and moving target detection based on MIMO synthetic aperture radar," Progress in Electromagnetics Research, vol. 131, pp. 311329, 2012.

[11] A. E. Waadt, S. Wang, C. Kocks et al., "Positioning in multiband OFDM UWB utilizing received signal strength," in Proceedings of the 7th Workshop on Positioning, Navigation and Communication (WPNC '10), pp. 308-312, March 2010.

[12] W. Z. Li, Z. Li, H. Lv et al., "A new method for non-line-ofsight vital sign monitoring based on developed adaptive line enhancer using low centre frequency UWB radar," Progress in Electromagnetics Research, vol. 133, pp. 535-554, 2013.

[13] I. C. Vizitiu, Fundamentals of Electronic Warfare, Matrix Rom Publishing House, 2011.

[14] K. Mizui, M. Uchida, and M. Nakagawa, "Vehicle-to-vehicle communication and ranging system using spread spectrum technique (Proposal of Boomerang Transmission System)," in Proceedings of the 43rd IEEE Vehicular Technology Conference, pp. 335-338, Secaucus, NJ, USA, May 1993.

[15] C. Sturm and W. Wiesbeck, "Waveform design and signal processing aspects for fusion of wireless communications and radar sensing," Proceedings of the IEEE, vol. 99, no. 7, pp. 12361259, 2011.

[16] B. J. Donnet and I. D. Longstaff, "Combining MIMO radar with OFDM communications," in Proceedings of the 3rd European Radar Conference (EuRAD '03), pp. 37-40, September 2006.

[17] CEPT Report 34: Report B from CEPT to European Commission in response to the Mandate 4 on Ultra-Wideband (UWB), 2009.

[18] European Communications Office, "Specific UWB applications in the bands $3.4-4.8 \mathrm{GHz}$ and $6-8.5 \mathrm{GHz}$ location tracking applications for emergency services (LAES), location tracking aplications 2 (LT2) and location tracking and sensors applications for automotive and transportation environments (LTA)," ECC Report 170, 2011.

[19] H. S. Rad and S. Gazor, "The impact of non-isotropic scattering and directional antennas on MIMO multicarrier mobile communication channels," IEEE Transactions on Communications, vol. 56, no. 4, pp. 642-652, 2008.

[20] A. M. Pistea and H. Saligheh Rad, "Three dimensional spacetime-frequency description of WB and UWB MIMO channels," IEEE Communications Letters, vol. 16, no. 2, pp. 205-207, 2012.

[21] A. M. Pistea, T. Palade, A. Moldovan, and H. S. Rad, "The impact of antenna directivity and channel bandwidth on the power spectral density of wideband and UWB MISO channels," in Proceedings of the 7th International Conference on Wireless and Mobile Communications (ICWMC '11), pp. 36-41, Luxembourg City, Luxembourg, June 2011.

[22] C. A. Balanis, Antenna Theory: Analysis and Design, John Wiley \& Sons, 2nd edition, 1996.

[23] M. Ghavami, L. B. Michael, and R. Kohno, Ultra Wideband Signals and Systems in Communication Engineering, John Wiley \& Sons, 2004.

[24] A. F. Molisch, K. Balakrishnan, D. Cassioli et al., "IEEE 802.15.4a channel model-final report," Technical Report Document IEEE 802.15-04-0662-02-004a, 2005.
[25] T. Kaiser and F. Zheng, Ultra-Wideband Systems with MIMO, John Wiley \& Sons, 2010.

[26] A. Abdi, J. A. Barger, and M. Kaveh, "A parametric model for the distribution of the angle of arrival and the associated correlation function and power spectrum at the mobile station," IEEE Transactions on Vehicular Technology, vol. 51, no. 3, pp. 425-434, 2002.

[27] J. Ren and R. G. Vaughan, "Spaced antenna design in directional scenarios using the von mises distribution," in Proceedings of the 70th IEEE Vehicular Technology Conference (VTC '09), pp. 1-5, Anchorage, Alaska, USA, September 2009.

[28] Q. Spencer, M. Rice, B. Jeffs, and M. Jensen, "A statistical model for angle of arrival in indoor multipath propagation," in Proceedings of the 47th IEEE Vehicular Technology Conference, vol. 3, pp. 1415-1419, Phoenix, Ariz, USA, May 1997.

[29] R. C. Qiu and I.-T. Lu, "Wideband wireless multipath channel modeling with path frequency dependence," in IEEE International Conference on Communications (ICC '96), Conference Record, Converging Technologies for Tomorrow's Applications, vol. 1, pp. 277-281, Dallas, Tex, USA, June 1996.

[30] J. P. M. de Sá, Applied Statistics Using SPSS, STATISTICA, MATLAB and R, Springer, 2003.

[31] J. Wallace, Characterization and identification of ultrawideband radio propagation channels [Ph.D. thesis], Faculty of the Graduate School, University of Southern California, 2005.

[32] A. F. Molisch, "Ultrawideband propagation channels-theory, measurement, and modeling," IEEE Transactions on Vehicular Technology, vol. 54, no. 5, pp. 1528-1545, 2005.

[33] M. C. Jeruchim, P. Balaban, and K. S. Shanmugan, Simulation of Communication Systems, Applications of Communications Theory, Plenum Press, New York, NY, USA, 1992.

[34] W. Q. Malik, "Spatial correlation in ultrawideband channels," IEEE Transactions on Wireless Communications, vol. 7, no. 2, pp. 604-610, 2008.

[35] D. Gesbert, H. Bölcskei, D. A. Gore, and A. J. Paulraj, "Outdoor MIMO wireless channels: models and performance prediction," IEEE Transactions on Communications, vol. 50, no. 12, pp. 19261934, 2002.

[36] P. Jin, N. A. Goodman, and K. L. Melde, "Exploiting directional antennas for reduced-dimension space-time RAKE receiving," IEEE Transactions on Vehicular Technology, vol. 57, no. 6, pp. 3880-3885, 2008.

[37] C. R. Anderson, "Low-antenna ultra wideband spatial correlation analysis in a forest environment," in Proceedings of the Vehicular Technology Conference, pp. 1-5, Barcelona, Spain, 2009.

[38] X. Cheng, C.-X. Wang, H. Wang et al., "Cooperative MIMO channel modeling and multi-link spatial correlation properties," IEEE Journal on Selected Areas in Communications, vol. 30, no. 2, pp. 388-396, 2012.

[39] X. Cheng, B. Yu, L. Yang et al., "Communicating in the real world: 3D MIMO," IEEE Wireless Communications Magazine, vol. 21, no. 4, pp. 136-144, 2014. 

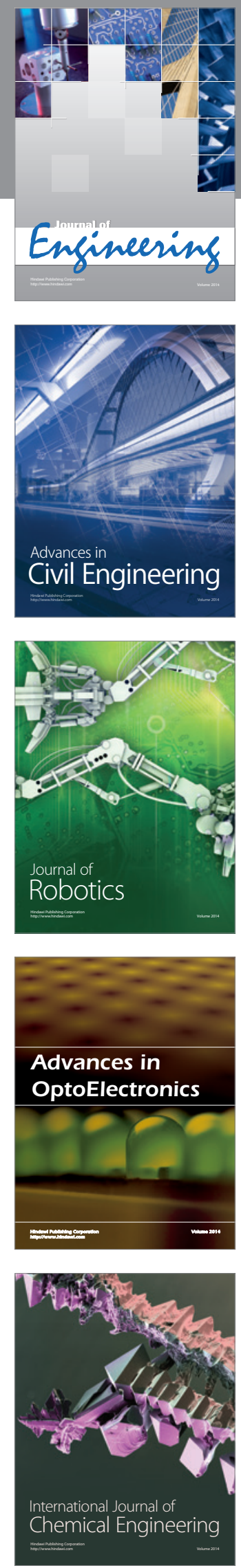

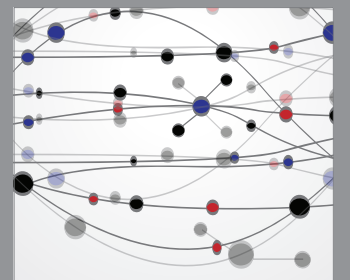

The Scientific World Journal
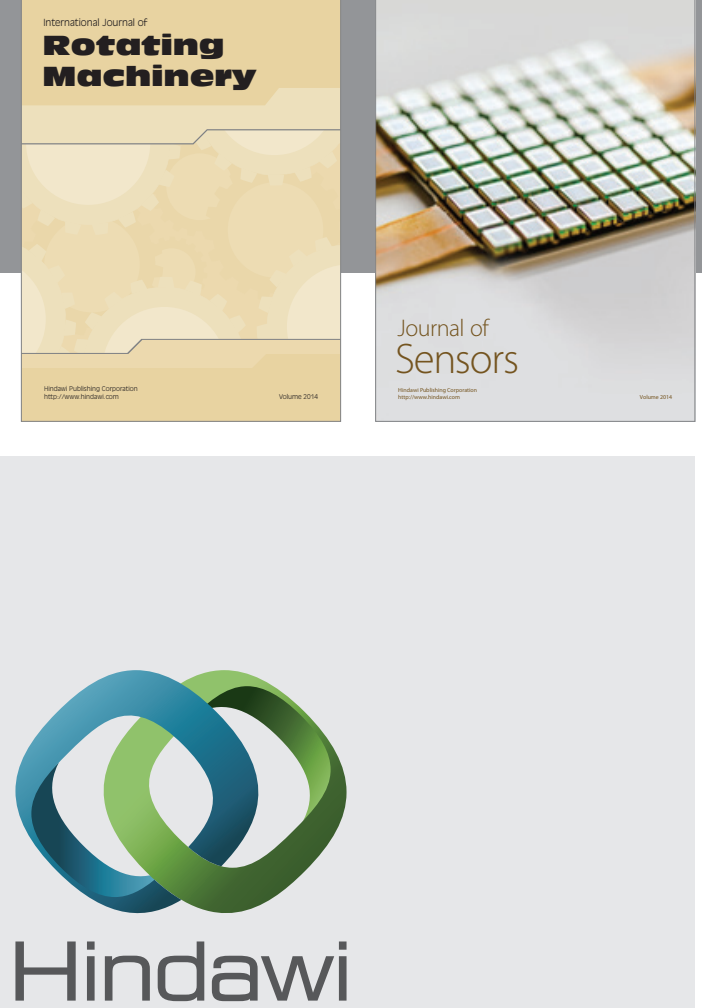

Submit your manuscripts at http://www.hindawi.com
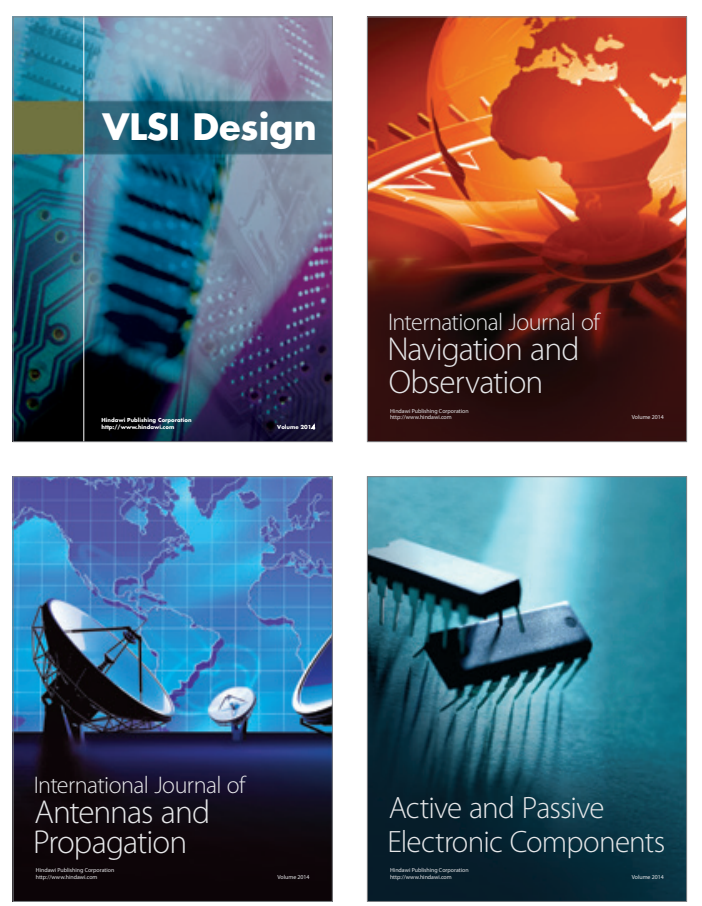
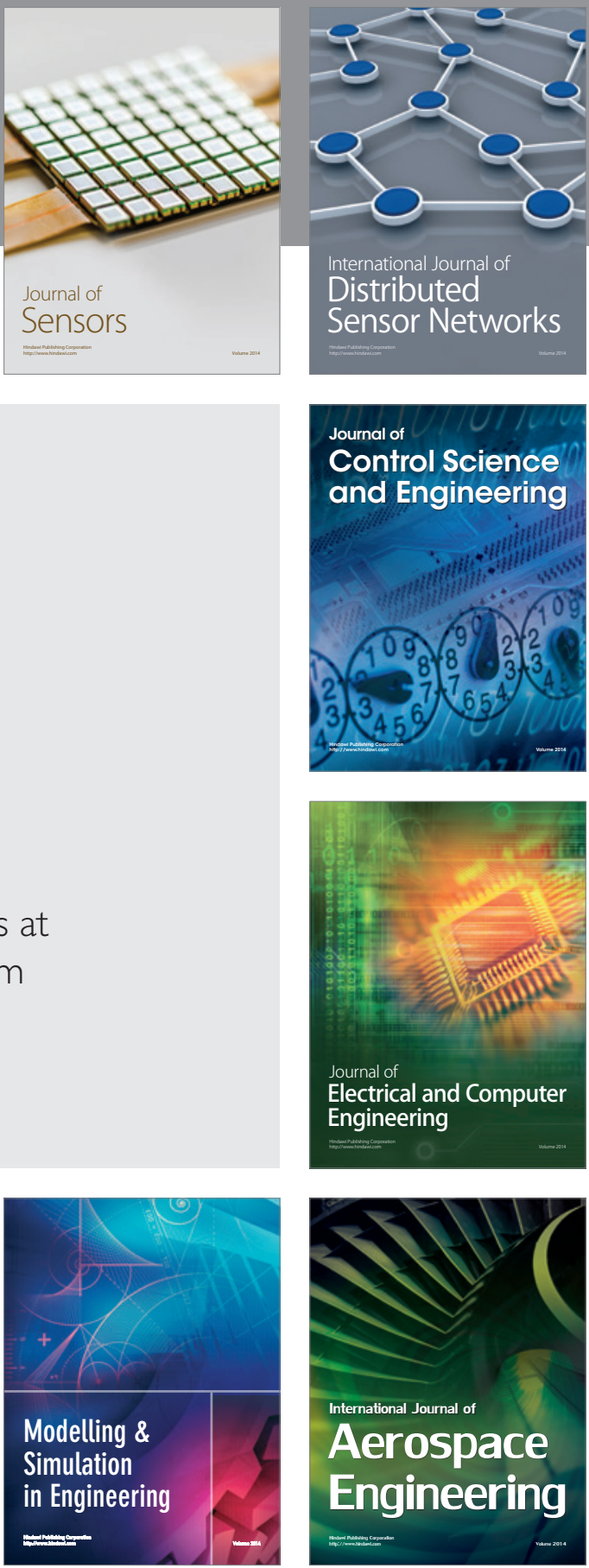

Journal of

Control Science

and Engineering
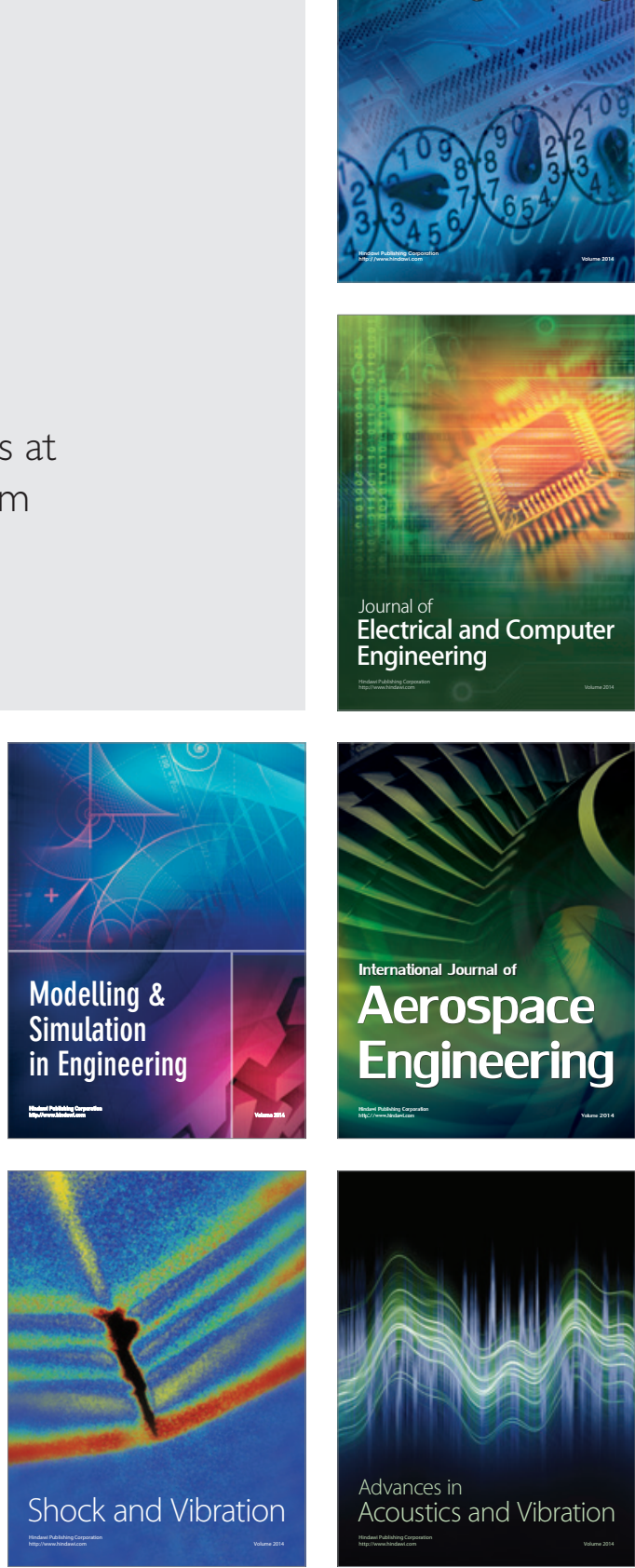